\title{
Activation of adenosine receptor A2a by gut symbionts promotes immune tolerance
}

\author{
Aleksandar Kostic ( $\nabla$ aleksandar.kostic@joslin.harvard.edu ) \\ Harvard Medical School https://orcid.org/0000-0002-0837-4360 \\ Tao Xu \\ Joslin Diabetes Center and Harvard Medical School \\ Lidan Zhao \\ Harvard Medical School \\ Md Zohorul Islam \\ Harvard University https://orcid.org/0000-0002-2015-5195 \\ Loc-Duyen Pham \\ Harvard Medical School \\ Martina Corsi \\ Harvard Medical School \\ Gregory Phillips \\ lowa State University \\ Michael Wannemuehler \\ lowa State University \\ Christophe Benoist \\ Harvard Medical School https://orcid.org/0000-0003-1172-6555
}

\section{Biological Sciences - Article}

Keywords: gut microbiome, immune regulation, autoimmune diseases, adenosine receptor, GPCR.

Posted Date: April 7th, 2021

DOl: https://doi.org/10.21203/rs.3.rs-376619/v1

License: @ (i) This work is licensed under a Creative Commons Attribution 4.0 International License.

Read Full License 


\section{Activation of adenosine receptor A2a by gut symbionts promotes}

2 immune tolerance

3

4 Tao Xu U $^{1,2}$, Lidan Zhao ${ }^{1,3}$, Md Zohorul Islam ${ }^{1,2}$, Loc-Duyen Pham ${ }^{1,2}$, Martina Sassone

5 Corsi $^{2}$, Gregory J. Phillips ${ }^{4}$, Michael Wannemuehler ${ }^{4}$, Christophe O. Benoist $^{2}$,

6 Aleksandar D. Kostic ${ }^{1,2^{*}}$

7

8 Affiliations:

91 Section on Pathophysiology and Molecular Pharmacology, Joslin Diabetes Center, 10 Boston, MA, USA

112 Department of Microbiology and Immunobiology, Harvard Medical School, Boston, 12 MA, USA

133 State Key Laboratory of Complex, Severe, and Rare Diseases, and Department of 14 Rheumatology and Clinical Immunology, Chinese Academy of Medical Sciences and 15 Peking Union Medical College, Beijing, China

164 Vet Microbiology \& Preventive Medicine, lowa State University, IA, USA

$17{ }^{*}$ corresponding author: Aleksandar.Kostic@joslin.harvard.edu

20 Keywords: gut microbiome, immune regulation, autoimmune diseases, adenosine 21 receptor, GPCR. 


\section{Abstract}

24 The gut microbiota is critical to immune homeostasis ${ }^{1-4}$, but our understanding of the

25 underlying molecular mechanisms is very limited. Here, we demonstrate a division of

26 labor among members of the eight-membered "model microbiome" altered Schaedler

27 flora ${ }^{5}$ in promoting distinct immunophenotypes. We report that Parabacteroides

28 goldsteinii ASF519 induces immune tolerance by promoting interleukin (IL)-10

29 production in a variety of myeloid-derived immune cells. The IL-10 induction is

30 dependent on the activation of adenosine receptor A2a by microbial enzymes of the

31 methionine cycle. ASF519 colonization in mice increased the level of adenosine in

32 ceca and induced IL-10 secreting dendritic cells in colonic lamina propria. These

33 immunophenotypes were pharmacologically reversed by A2a blockage. In mouse

34 models of human autoimmune diseases, ASF519 supplementation significantly

35 ameliorated insulitis in type 1 diabetes and collagen-induced arthritis. This study

36 unveils a novel paradigm of gut microbiota-adenosine receptor interactions in

37 immune tolerance and potentially provides a new therapeutic strategy for immune

38 disorders.

39

40 


\section{Introduction}

42 Previous studies have demonstrated reciprocal interactions between the immune

43 system and the gut microbiota ${ }^{1,6,7}$. The importance of the gut microbiota to immune

44 function has been demonstrated by a wide range of immune functional and structural deficiencies in germ-free (GF) or antibiotic-treated mice ${ }^{2-4}$ that can be rescued by microbial recolonization in the gut. We now appreciate that gut microbial dysbiosis caused by genetic susceptibility, antibiotic use, or Westernized diet, for example, can influence intestinal diseases, immune disorders, cancer, and metabolic syndromes ${ }^{7}$. However, it is necessary to move from microbiome-wide association studies to causal microbe identification, and ultimately to the underlying molecular mechanisms to fully exploit the microbiome for therapeutic intervention. Currently, our knowledge of microbial immunomodulation is mainly mechanistically limited to activation of Tolllike receptors (e.g., TLR2/4/6), members of G protein-coupled receptors (GPCR 41/43/109a, P2XR, and TGR5), and nuclear receptors (AhR, PXR, FXR), or inhibition of histone deacetylase in immune cells, by microbially derived polysaccharides, short-chain fatty acids, ATP, and bile acids, and amino acid derivatives ${ }^{8}$.

Synthetic microbial consortia consisting of known members retain a lower complexity of inter-microbial and microbe-host interactions than natural gut microbiota but still show tremendous immunological and ecological impacts in the gut $^{9}$. Examples include the 17 -member Treg inducing Clostridia consortium ${ }^{10}$, the 11member IFNy+ CD8+ T cell inducing bacterial assembly ${ }^{11}$, and the multi-functional 8member altered Schaedler flora $(\mathrm{ASF})^{5}$. The immunomodulatory effects of gut microbes appear independent of microbial phylogeny ${ }^{12}$. The ASF is genetically competent in terms of gene richness ${ }^{13}$ and its wide phylogenetic distribution (Extended Data Fig. 1a), and more importantly, it can resolve immunological, neurological, and metabolic defects in germ-free mice ${ }^{14-22}$. It increases IL-10 production and promotes Treg proliferation in the colon ${ }^{16}$ and is able to mimic the specific pathogen-free microbiota in reducing insulitis in $M y D 88^{-/-}$NOD mice ${ }^{23}$. These results indicate a pronounced immune tolerance-eliciting function of this defined community, but the identity of the responsible microbes and molecules is yet undetermined. Here, we dissected the role of each individual ASF member in a panel of immune regulation assays and elucidated a novel microbial immunomodulatory 
mechanism involving the adenosine receptor A2a. These functional microbiome studies have the potential to open novel therapeutic avenues for the prevention and treatment of immune diseases.

\section{Results}

\section{Division of labor among murine intestinal microbes in in vitro immune} regulation

Dendritic cells (DCs) are professional antigen-presenting cells that play a critical role in inducing immunity and maintaining immune tolerance. Whether they are tolerogenic or immunogenic mainly depends on their cytokine production profiles and impact on T cells. We interrogated the immunoregulatory potential of each ASF member by stimulating bone marrow-derived dendritic cells (BMDCs) with filtersterilized spent medium (SSM) collected during bacterial growth (Extended Data Fig. 1b). We found that SSM from Parabacteroides goldsteinii ASF519 dramatically induced the production of the anti-inflammatory cytokine IL-10 in a bacterial growthdependent manner (Fig. 1a; Extended Data Fig. 1c). In contrast, Clostridium spp. ASF356 only induced IL-10 at the exponential stage, while a weak IL-10 induction was observed with Eubacterium plexicaudatum ASF492 and Pseudoflavonifractor spp. ASF500. Measurement of another anti-inflammatory cytokine, TGF- $\beta$, revealed that Lactobacillus intestinalis ASF360 and Lactobacillus murinis ASF361 induced BMDCs to release this cytokine (Fig. 1a), which positively correlated with bacterial growth (Extended Data Fig. 1c); ASF519 had a mild effect on TGF- $\beta$. For their immunogenic potential, although the uninoculated bacterial culture medium induced a high level of pro-inflammatory TNF- $\alpha$, this effect was diminished by the monoculture of 7 ASF members (Fig. 1a), with the exception of E. plexicaudatum ASF492 that displayed a recurring TNF- $\alpha$ release when the stationary-phase SSM was used. These measurements revealed distinct cytokine inducing profiles of ASF members coupled with growth phase-specific microbial physiology (Fig. 1a). Notably, ASF519 is the only member that induced two anti-inflammatory cytokines, IL-10 and TGF- $\beta$ (Fig. 1b).

We then tested the impact of exposed BMDCs on Treg proliferation via in vitro coculture with splenocytes from conventional mice (Extended Data Fig. 1b). Interestingly, BMDCs exposed to ASF519 SSM, especially collected from late 
exponential and stationary phases, strongly promoted Foxp3+ Treg proliferation

107 ( $80 \pm 6 \%$ by ASF519 SSM versus $20 \pm 9 \%$ by uninoculated bacterial culture medium)

108 (Fig. 1a, Extended Data Fig. 1d). The Foxp3+ Treg subset increased 1.8-fold when

109 cocultured with the ASF519 SSM-stimulated BMDCs (Fig. 1c). A weak stimulatory

110 effect on Treg proliferation was observed with three other members (ASF356,

111 ASF361, and ASF502) but the effect peaked at distinct bacterial growth stages. Their

112 impacts on both cytokine profiles and Treg proliferation demonstrate a "division of

113 labor" among ASF members in regulating immunophenotypes of BMDCs (Fig. 1b).

114 In particular, ASF519 promotes tolerogenic phenotypes of BMDCs via inducing anti-

115 inflammatory cytokines and Treg proliferation. Considering the high abundance of

116 ASF519 in the feces of ASF mice ${ }^{5}$ and the relatively high prevalence ( 23\%) of $P$.

117 goldsteinii in the human gut microbiome according to The Human Microbiome

118 Project (Extended Data Fig. 2), we were compelled to further explore its

119 immunoregulatory activities.

120 Synergistic regulation of myeloid cell-specific IL-10 induction

121 Because IL-10 is produced by a variety of immune cell types and is involved in both 122 innate and adaptive immunity, we tested the IL-10 inducing specificity of ASF519 on

123 a panel of diverse immune cells. We found that stimulation of isolated DCs, bone 124 marrow-derived macrophages, and isolated splenic monocytes all yielded a high 125 level of IL-10 (Fig. 1d); however, neither splenic panB nor panT stimulation, nor 126 stimulation of immune-irrelevant BEND3 endothelial cells, showed a significant 127 impact on IL-10 production. These data suggest that ASF519-mediated IL-10 128 induction is specific to myeloid-derived antigen-presenting cells. We also observed 129 that IL-10 induction is independent of TNF-a signaling (Extended Data Fig. 3a), and 130 that it is a slow response, which ramps up after $24 \mathrm{hrs}$ of monotonous stimulation of 131 BMDCs by ASF519 SSM (Extended Data Fig. 3b and 3c).

132 RNA sequencing for BMDCs revealed dramatic transcriptomic changes 133 caused by stimulation with ASF519 SSM versus broth control (adjusted $p<0.01$, |fold 134 change|>3) (Fig. 1e and 1f, Extended Data Fig. 4a and 4b). Further stimulation by 135 zymosan induced significant effects in broth-treated rather than ASF519 SSM-

136 treated cells (Extended Data Fig. 4b-4d), suggesting that ASF519 SSM may 137 increase the tolerance of BMDCs to immunogenic zymosan. ASF519-induced 138 differentially expressed genes have a wide association with the regulation of immune 
140 Data Fig. 4e). Notably, negative regulation of antigen processing and presentation of

141 peptide or polysaccharide antigen via MHC class II, and negative regulation of

142 oligodendrocyte apoptotic process, are enriched most in upregulated and

143 downregulated gene sets, respectively. At the gene level (Fig. 1g, Extended Data

144 Fig. 4f), we observed a dramatic induction of tolerogenic $/ / 10$, lipocalin-2 (Lcn2), and

145 indoleamine 2, 3-dioxygenases (Ido1/2), as well as a reduction of the co-stimulatory

146 molecule Cd40 and inflammatory cytokines, including I/12b, I/23a, and I/1f9 (or II-36

147 gamma). It is known that IL-36 gamma promotes inflammation and DC maturation;

148 IL-12B and IL-23A together build up the heterodimeric inflammatory IL-23 which is

149 associated with autoimmune diseases and cancer. Their decreased transcript

150 abundance may additively enhance the tolerogenic phenotypes of BMDCs. It is also

151 notable that this step-wise stimulation with ASF519 SSM added before zymosan

152 exerted a synergistic and antagonistic effect on certain transcripts (Fig. 1g,

153 Extended Data Fig.4g-4j). For instance, I/10, Ido2, and U90926 were synergistically

154 induced; whereas suppression of $/ / 12 b$, $/ / 1 f 9$, and Cd40 by ASF519 SSM was

155 attenuated by subsequent zymosan stimulation. We validated this synergy on //10 at

156 the protein level (Fig. 1h). Therefore, components of ASF519 SSM can induce

157 multifaceted tolerogenic phenotypes of BMDCs in vitro.

158 Microbial activation of adenosine receptor A2a induces IL-10 production

159 To further dissect this immunomodulatory mechanism, we sought to identify the 160 responsible molecules using bioanalytical techniques (Fig. 2a). The IL-10 inducing

161 function of ASF519 SSM was largely heat-labile (Extended Data Fig. 5a) and 162 immobilized proteinase K digestion of ASF519 SSM completely abolished IL-10

163 induction (Fig. 2b). These results, plus a dose-dependent IL-10 induction by

164 concentrated crude proteins (Fig. 2c), confirmed the proteinaceous nature of the

165 responsible molecules. We then performed gel filtration to fractionate crude proteins

166 in ASF519 SSM. BMDC assays revealed consecutive fractions of an early elution

167 peak exhibiting strong IL-10 induction (Fig. 2d). The elution chromatogram

168 demonstrated that these bioactive fractions were in the excluded volume of the gel

169 filtration column that was designed for separating 20-8,000 kDa globular proteins

170 (Extended Data Fig. 5b-5c), suggesting a large structure is formed. Native- and

171 SDS-PAGE analysis of these bioactive fractions confirmed the existence of a 
complex architecture that is susceptible to proteinase $\mathrm{K}$ digestion (Extended Data

173 Fig. 5d). Mass spectrometry analysis of major gel bands identified proteins

174 associated with cysteine and methionine metabolism, including

175 adenosylhomocysteinase (AHCY), O-acetyl-L-serine sulfhydrylase (OASS),

176 methionine adenosyltransferase (MAT), and O-acetylhomoserine sulfhydrylase

177 (METY). Because the integrity of proteinaceous factors is essential for IL-10

178 induction, we speculated metabolites of these enzymes are the actual effectors,

179 which are naturally heat-labile or dependent on substrate availability. This hypothesis

180 is supported by the known immunosuppressive role of naturally occurring

181 nucleosides in mammals, such as adenosine, inosine, and 5'-methylthioadenosine

182 (MTA), which can also stem from bacterial methionine metabolism and purine

183 metabolism. These nucleosides activate membrane adenosine receptors (A1, A2a,

$184 \mathrm{~A} 2 \mathrm{~b}$, and $\mathrm{A} 3$ ) on a variety of immune cells to promote tolerogenic or anti-

185 inflammatory phenotypes ${ }^{24}$.

To test whether ASF519-mediated IL-10 induction is mediated by the activation of adenosine receptors, we applied adenosine receptor-specific antagonists to BMDC assays. Intriguingly, supplementation of the A2a inhibitor ZM241385 drastically dampened IL-10 induction in a dose-dependent manner in response to ASF519 SSM (Fig. 2e). By contrast, antagonists of A1, A2b, and A3 at the tested concentrations had no significant impact (Fig. 2e). The engagement of A2a in IL-10 induction was pharmacologically reproduced using bioactive gelfiltration fractions as stimuli of BMDCs in inhibition assays (Extended Data Fig. 5e) and further confirmed with the genetic knockout of A2a where A2a ${ }^{-/-}$BMDCs disabled IL-10 induction when exposed to ASF519 SSM (Fig. 2f). A2a activation then increased the intracellular level of cyclic AMP (Extended Data Fig. 5f), which is known to be involved in the transcriptional regulation of //10. Therefore, activation of adenosine receptor $\mathrm{A} 2 \mathrm{a}$ signaling in BMDCs is required for IL-10 induction by microbial proteins.

AHCY can hydrolyze S-adenosylhomocysteine (SAH) to generate adenosine, a natural ligand of adenosine receptors. To validate its role in IL-10 induction, we first applied the AHCY inhibitor DZA to BMDC assays, which revealed that AHCY inhibition decreased IL-10 induction in a dose-dependent manner (Fig. 2e). We then heterologously expressed and purified recombinant enzymes (Extended Data Fig.

205 6a-6d), including AHCY, OASS, MAT, METY, and methionine synthase (MS), for in 
vitro enzymatic and immunological assays. As expected, only purified AHCY demonstrated the enzymatic activity of using SAH as a substrate to produce adenosine (Fig. 2g). Using purified AHCY to stimulate BMDCs successfully induced IL-10 in a dose-dependent manner (Fig. 2h). Apart from AHCY, two other enzymes of the methionine cycle (MS and MAT) were also able to induce IL-10 at different optimal concentrations (Fig. 2h). Because OASS and METY are involved in cysteine metabolism and irrelevant to IL-10 induction, we conclude that enzymatic activities of the ASF519-derived methionine cycle contribute to IL-10 induction in BMDCs (Fig. 2i).

\section{ASF519 colonization induces tolerogenic phenotypes}

To probe in vivo immunoregulatory function, ASF519 was orally introduced to 4week old germ-free mice for a 2-week long mono-association. Untargeted metabolomics revealed vast changes in the abundance of cecal metabolites caused by ASF519 mono-association (Extended Data Fig. 7a). These metabolites were significantly enriched for the metabolism of 13 amino acids, galactose, and amino and nucleotide sugars (Fig. 3a, extended Data Fig. 7b). Notably, ASF519 colonization increased cecal adenosine by 15 -fold, as well as the adenosine-forming substrate SAH and one of the adenosine-degradation products, adenine, by 9 -fold and 73-fold, respectively (Fig. 3b). Therefore, ASF519 increases the presence of adenosine and related metabolites in cecal contents of mice.

With qPCR analysis for 34 select genes involved in intestinal integrity, mucin synthesis, and immune functions, we found ASF519 mono-association increased the transcript level of $/ / 10$ in the small intestine and colon and I/17a in the colon, and it significantly reduced $/ / 5$ transcripts in the small intestine (Fig. 3c). //10 up-regulation is consistent with ASF519 SSM-mediated IL-10 induction observed in vitro. Other genes involved in mucin synthesis and intestinal integrity were not influenced by ASF519, with the exception of an increase in the cell proliferation promoting myc gene in the small intestine (Extended Data Fig. 8). Measurement of 18 cytokines in sera revealed a systematic reduction of IL-4 and IL-5 in ASF519 mice (Fig. 3d and 3e). IL-5 is mainly secreted by activated T helper 2 cells which originally rely on IL-4 to differentiate from naive T cells, and both IL-4 and IL-5 are involved in the proliferation and function of activated $B$ and $T$ cells. Their concurrent reduction in circulation may imply the effects of ASF519 on systemic immune response. 

immunoregulation in vivo, we blocked $A 2 a$ signaling by i.p. injection of an $\mathrm{A} 2 \mathrm{a}$ antagonist, ZM 241485, into antibiotic-pretreated mice that received live or heatkilled ASF519 (Fig. 3f). Immunophenotyping of colonic lamina propria cells revealed an increase in IL-10 producing DCs among CD103- CD11C+ and CD103+ CD11C+ populations in live versus killed ASF519 recipients (Fig. $\mathbf{3 g}$ and $\mathbf{3 h}$ ). Importantly, these effects were significantly decreased by the A2a antagonist. This IL-10 immunophenotype is consistent with our in vitro observation that blockage of $\mathrm{A} 2 \mathrm{a}$ rather than other adenosine receptors sufficiently interrupted IL-10 production by BMDCs in response to ASF519 SSM. Other major immune cell types, such as CD11b+F4/80+ macrophages, CD4+ T cells, and CD19+ CD45+ B cells, did not show significant changes in their IL-10 secretion (Extended Data Fig. 9 and 10). It is also notable that ASF519-treated mice exhibited decreased lamina propria B cells and CD11b+ CD45+ cells in the colon (Fig. $\mathbf{3 i}$ and $\mathbf{3 j}$ ), which was also restored by A2a blockage. Together, these data demonstrate that the immunoregulatory function of ASF519 in colonic lamina propria goes beyond IL-10 induction in DCs to include more general homeostatic activities of leukocytes through A2a activation.

\section{Oral administration of ASF519 ameliorates autoimmune disorders}

Type 1 diabetes (T1D) is an autoimmune disease in which insulin-producing beta cells in the pancreas are destroyed by T cells. DCs promote the priming and effector differentiation of autoreactive T cells in the pancreatic lymph nodes ${ }^{25}$. To test if the immunoregulatory functions of ASF519 can curb the immunopathogenesis in T1D, we introduced ASF519 to T1D-prone non-obese diabetic mice (NOD) by weekly oral gavage from four to thirteen weeks of age (Fig. 4a). At 14 weeks, ASF519 recipients had a lower incidence of hyperglycemia (>250 mg/dL, 57\%) compared to killed ASF519 recipients (88\%) (Fig. 4b), indicating an improved pancreatic function as a result of ASF519 supplementation. To measure immunopathogenesis directly in the pancreas, mice were sacrificed at 14 weeks old to perform histological analysis of insulitis by scoring infiltration of insulin-producing beta cells by pathogenic lymphocytes. In line with improved glucose control, the severity of insulitis was reduced in ASF519 mice (Fig. 4c). Insulin immunostaining on adjacent sections of histological staining also demonstrated that more insulin-producing beta cells were 
271 present in the islets of ASF519 mice. Therefore, ASF519 colonization in NOD mice

272 plays a protective role against T1D progression.

273 We then asked whether ASF519 could ameliorate other autoimmune

274 diseases. Rheumatoid arthritis is an autoimmune disease that mainly involves the

275 synovial tissues within joints. We introduced ASF519 to DBA mice before the

276 injection of collagen to induce arthritis (Fig. 4d). The qPCR analysis confirmed the

277 long-term colonization of ASF519 in the gut of DBA mice as after 3 weeks of the final

278 gavage, the abundance of ASF519 in feces was $>11$ fold higher in live versus killed

279 ASF519 recipient mice (Fig. 4e). Following 30 days post-collagen immunization,

280 mice that received killed ASF519 significantly lost body weight (Fig. 4f), whereas

281 ASF519 mice were able to maintain their body weight. In line with that, killed ASF519

282 recipient mice developed much more severe arthritis than ASF519 mice (Fig. $\mathbf{4 g}$ ).

283 These data demonstrate that ASF519 colonization strongly protected DBA mice from

284 collagen-induced arthritis.

\section{Discussion}

286 Accumulating evidence from mouse and human studies demonstrates that the

287 microbiome is integral to immune regulation, and that alterations to the microbiome

288 may underlie many immune-mediated and metabolic diseases. Here, we leverage

289 the ASF, an important and long-studied "model microbiome" to demonstrate that

290 there exists a division of labor among members of the microbiome in modulating

291 immune regulatory phenotypes. This led us to identify that one specific member, $P$.

292 goldsteinii ASF519, is a potent inducer of IL-10 in myeloid cells in an adenosine

293 receptor A2a-dependent manner both in vitro and in vivo. By a combination of

294 activity-guided fractionation and genetic methods, we identified that enzymes

295 belonging to the methionine/adenosine cycling pathway were sufficient to induce IL-

29610 in dendritic cells. Furthermore, gnotobiotic mice mono-colonized with ASF519

297 exhibited a profound increase in cecal abundance of adenosine, adenine, and S-

298 adenosylhomocysteine. This evidence combined with our data showing that the IL-

29910 induction is A2a-dependent demonstrates that the IL-10-inducing activity of

300 ASF519 is mediated by adenosine signaling. Finally, we demonstrated that ASF519

301 supplementation can reduce the severity of arthritis in the CIA model as well as

302 insulitis resulting from autoimmune diabetes in NOD mice. 
The novel mechanism we here describe is distinct from known microbially

304 involved IL-10 regulation, including IL-10 induction in DCs, macrophages, or

305 monocytes by bacterial polysaccharide capsules ${ }^{26-27}$, and SCFA-mediated induction

306 through GPCR43 activation in microbiota antigen-specific Th1 cells ${ }^{28}$. Our study

307 broadens the previously described GPCR interactions with gut microbial

308 metabolites ${ }^{29,30}$. In regard to the critical role of DCs in both innate and adaptive

309 immunity, the increased IL-10 induction by ASF519 promotes mucosal immune

310 tolerance by reducing antigen presentation to lymphocytes and the immunogenic

311 potency of leukocytes in colonic lamina propria. As A2a signaling is not only involved

312 in immunomodulation, but also metabolism ${ }^{31}$ and cell proliferation ${ }^{32}$, ASF519

313 mediated A2a activation may modulate multiple biological functions that together

314 manifest the observed benefits in disease models here described.

315 This work serves as a model workflow by which future immune phenotypes

316 can be screened in the ASF system in vitro and then interrogated in vivo towards the

317 discovery and characterization of novel immunoregulatory functions of the gut

318 microbiome. Furthermore, this workflow may in the future help formulate

319 combinatorial strategies to sharpen the regulatory potency of microbes or their

320 secreted products for therapeutic use. Supplementation with ASF519 or potentially

321 related Parabacteroides species may represent a novel strategy to prevent and/or

322 treat autoimmune and inflammatory diseases. 


\section{Animal models}

$327 \mathrm{C} 57 \mathrm{BL} / 6,129 \mathrm{~S} 1 / \mathrm{J}, 1 / 10^{-/-}, A 2 \mathrm{a}^{-{ }^{-}}$, DBA/1J, NOD mice were purchased from Jackson

328 Laboratories and maintained in specific pathogen-free conditions. The $\mathrm{A}^{2 \mathrm{a}^{-/-}}$mice were on a mixed genetic background of BALB/c and 129S. Unless otherwise indicated, 8-12-week-old mice of both genders were used for in vitro experiments. Only male DBA and female NOD mice were used for disease studies. This study complied with all ethical regulations involving experiments with mice, and all experimental manipulations were performed in accordance with protocols approved by the Animal Care and Use Committee of Joslin Diabetes Center.

To study the in vivo function of Parabacteroides goldsteinii ASF519 in mice, we introduced this strain into 4-wk old both male and female germ-free (GF) mice by oral gavage of $200 \mu \mathrm{l}$ bacterial cells with $1 \times 10^{\wedge} 9 \mathrm{CFU}$ in PBS. The control GF group was orally administered with $200 \mu$ PBS. The SPF mice were developed by introducing fecal microbiota of conventional mice to GF mice. After 2 weeks of colonization, we sacrificed mice to collect sera, intestinal tissues, and cecal contents. Shotgun metagenomic sequencing for fecal and cecal samples confirmed monocolonization. Approximately, $1 \mathrm{~cm}$ of middle colon and ileum $(5 \mathrm{~cm}$ upstream of the cecum) were preserved in $0.5 \mathrm{ml} \mathrm{TRIZOL}$ buffer for storage at $-80^{\circ} \mathrm{C}$.

We tested the engagement of adenosine receptor A2a in ASF519-mediated immune regulation through pharmacological blockade in mice. 9-11-wk old C57BL/6J mice were treated by an antibiotic cocktail $(0.5 \mathrm{~g} / \mathrm{L}$ vancomycin, $1.36 \mathrm{~g} / \mathrm{L}$ neomycin, plus $3.75 \mathrm{~g} / \mathrm{L}$ sweeter aspartame) for $48 \mathrm{hrs}$. Then, the antibiotic water was exchanged with a $10 \%(\mathrm{w} / \mathrm{w})$ PEG4000 solution in water, and the mice were fasted for $16 \mathrm{hrs}$. After that, we administered these prepared mice twice at the interval of 3 days by gavaging $200 \mu$ of live or heat-killed $P$. goldsteinii in PBS at $1 \times 10^{\wedge} 9 \mathrm{CFU}$. Both live and killed $P$. goldsteinii recipient groups were further divided with a daily intraperitoneal injection of $100 \mu \mathrm{l}$ A2a antagonist ZM $241385(1.25 \mathrm{mg} / \mathrm{ml})$ or vehicle (7.4\% DMSO + 7.4\% Cremophor + 85.2\% PBS) for 17 days. We collected stool samples at 5 timepoints: before-antibiotic treatment, post-antibiotic/pre-10\% PEG4000 treatment, post-gavage day 1, day 3, and day 7 . On post-gavage day 14 , we harvested colon tissues and cecal contents for immunophenotyping and metabolomic analysis, respectively. 
To test whether $P$. goldsteinii influences the development of autoimmune

359

360

361

362

363

364

365

366

367

368

369

370

371

372

373

374

375

376

377

378

379

380

381

382

383

384

385

386

387

388

389

390 diabetes, we introduced this bacterium into non-obese diabetic mice (NOD) which are prone to develop insulitis and diabetes ${ }^{33}$. 4-wk old female NOD mice received $200 \mu \mathrm{l}$ of live or heat-killed ASF519 in PBS at approximately $1 \times 10^{\wedge} 9$ CFU by weekly oral administration until 13-wk old. Blood glucose was measured in tail tip blood at 14 weeks. The blood glucose at $>250 \mathrm{mg} / \mathrm{dL}$ defines hyperglycemia ${ }^{34}$. We collected the pancreas for histological studies, including Hematoxylin and Eosin staining and insulin immunostaining.

We also tested the protective role of $P$. goldsteinii in the collagen-induced arthritis mouse mode ${ }^{35}$, which is the most commonly studied autoimmune model of rheumatoid arthritis. 10-wk old male DBA/1J mice were orally administered with 200 $\mu \mathrm{l}$ of live or heat-killed ASF519 in PBS at $1 \times 10^{\wedge} 9$ CFU for twice a week in a course of two weeks. After microbial colonization, mice were immunized by $50 \mu \mathrm{l}$ of $1 \mathrm{mg} / \mathrm{ml}$ collagen emulsion in Complete Freund's Adjuvant (CFA, Sigma-Aldrich \#F5506$10 \mathrm{ML}$ ) that consists of $1 \mathrm{mg} / \mathrm{ml}$ of heat-killed Mycobacterium tuberculosis (Invitrogen \#tlrl-hkmt-1) by subcutaneous injection at about $1.5 \mathrm{~cm}$ distal from the base of the tail. This formulation in CFA has been demonstrated to be essential to induce arthritis in mice from the autoimmune response that results in arthritis of major joints within 4-6 weeks of injection. We monitored mice twice weekly for arthritis and bodyweight, assessed arthritis severity by the established qualitative scoring system through observing and measuring the severity of paw inflammation ${ }^{35}$. Each paw was evaluated and scored individually on a scale of $0-4$, with 4 indicating the most severe inflammation. The maximal combined score from 4 paws per mouse is 16 .

\section{Collection of bacterial spent media}

ASF isolates were provided by Dr. Michael Wannemuehler at lowa State University. We grew each ASF member in the brain heart infusion broth (BHI) under the anaerobic condition at $37^{\circ} \mathrm{C}$. The BHI medium consists of $37 \mathrm{~g} / \mathrm{L} \mathrm{BHI}$ (Sigma), $5 \mathrm{~g} / \mathrm{L}$ yeast extract, $0.5 \mathrm{~g} / \mathrm{L} \mathrm{L}$-cysteine, $10 \mathrm{mg} / \mathrm{L}$ resazurin, and $5 \mathrm{mg} / \mathrm{L}$ hemin, dissolved in $1 / 2(\mathrm{v} / \mathrm{v})$ miniQ water and $1 / 2(\mathrm{v} / \mathrm{v})$ tap water. After autoclaving, $1 \mathrm{~L}$ of $\mathrm{BHI}$ was supplemented with $0.2 \mathrm{ml} 1 \%$ vitamin $\mathrm{K}, 50 \mathrm{ml}$ newborn calf serum, and $50 \mathrm{ml}$ horse serum, and $10 \mathrm{ml}$ of $20 \%(\mathrm{~m} / \mathrm{v})$ filter-sterilized yeast extract. The medium was placed in the anaerobic chamber overnight to reduce oxygen before bacterial inoculation. The spent media were harvested at indicated time points during growth and then 
391 filter-sterilized twice with $0.2 \mu \mathrm{M}$ filters before storage at $-80^{\circ} \mathrm{C}$. Bacterial cell biomass

392 was estimated by monitoring OD600.

393 The defined medium used to grow ASF519 contains salts, carbon sources,

394 and supplements. The salts and carbon sources include $13.6 \mathrm{~g} / \mathrm{L} \mathrm{KH}_{2} \mathrm{PO}_{4}, 0.875 \mathrm{~g} / \mathrm{L}$

$395 \mathrm{NaCl}, 1.125 \mathrm{~g} / \mathrm{L}\left(\mathrm{NH}_{4}\right)_{2} \mathrm{SO}_{4}, 5.0 \mathrm{~g} / \mathrm{L}$ glucose, and $0.25 \mathrm{~g} / \mathrm{L} \mathrm{L}$-cysteine, pH7.2. $1 \mathrm{~L}$ of

396 the salts/carbon sources was then supplemented with $0.5 \mathrm{ml}$ of $10 \mathrm{mg} / \mathrm{ml}$ hemin, 200

$397 \mu \mathrm{l}$ of $1 \%$ vitamin $\mathrm{K}, 1.0 \mathrm{ml}$ of $0.1 \mathrm{M} \mathrm{MgCl}_{2}, 1.0 \mathrm{ml}$ of $0.4 \mathrm{mg} / \mathrm{ml} \mathrm{FeSO}{ }_{4} 7 \mathrm{H}_{2} \mathrm{O}, 1.0 \mathrm{ml}$ of

$3980.8 \% \mathrm{CaCl}_{2}, 10 \mathrm{ml}$ ATCC Vitamin complex, and $10 \mathrm{ml}$ ATCC mineral complex. The

399 medium was sterilized with $0.2 \mu \mathrm{m}$ filters and oxygen-reduced in an anaerobic

400 chamber before use.

401 Cell isolation and cultivation

402 We developed bone marrow-derived dendritic cells (BMDCs) from mouse bone

403 marrow. Mice at 8-12 wk old were euthanized and sacrificed to collect hind legs.

404 After removing skin, flesh, and muscles from the legs, bone marrow was flushed out

405 from bones and collected for red blood cell lysis using the RBC lysis buffer

406 (Invitrogen \#00-4333-57) by following the manufacturer's instruction. After

407 centrifugation at $500 \mathrm{~g}$ for $8 \mathrm{~min}$ at $4^{\circ} \mathrm{C}$, we resuspended hematopoietic cells in

408 complete DMEM (cDMEM) consisting of $10 \%$ fetal calf serum, $1 \times$ penicillin-

409 streptomycin solution (100x, Caisson Labs \#PSL01), 10 mM HEPES, and 5 M 2-

410 mercaptoethanol in Gibco DMEM medium (Thermo fisher \#10-566-024). To develop

411 BMDCs, hematopoietic cells were cultured in cDMEM with $20 \mathrm{ng} / \mathrm{ml}$ GM-CSF (2 x

$41210^{6}$ cells in $10 \mathrm{ml}$ per untreated petri dish $100 \mathrm{~mm} \times 15 \mathrm{~mm}$ ) at $37^{\circ} \mathrm{C}, 5 \% \mathrm{CO}_{2}$. The

413 supplemented medium was refreshed on days 3 and 6. BMDCs were collected on

414 day 9 and resuspended in the CryoStor CS10 buffer (STEMCELL \#07930) for

415 storage at $-80^{\circ} \mathrm{C}$.

416 To develop BM-DMs ${ }^{36}$, hematopoietic cells from bones were transferred into

417 culture-treated dishes (on average 1 leg per plate) for incubation at $37^{\circ} \mathrm{C}$ for $4 \mathrm{hrs}$ to

418 allow resident macrophage to adhere to the plastic. Cells in the supernatants were

419 collected and cultured in CDMEM with $10 \mathrm{ng} / \mathrm{ml} \mathrm{M-CSF}\left(2 \times 10^{6}\right.$ cells in $10 \mathrm{ml}$ per

420 culture treated dish $100 \mathrm{~mm} \times 15 \mathrm{~mm}$ ) at $37^{\circ} \mathrm{C}, 5 \% \mathrm{CO}_{2}$, and the medium was

421 refreshed every 3 days. BM-DMs were collected on day 10 for downstream

422 experiments. 
The BEND3 endothelial cell line was cultured in CDMEM without using GM-

424 CSF or M-CSF $\left(5 \times 10^{5}\right.$ cells in $10 \mathrm{ml}$ per culture treated dish $\left.100 \mathrm{~mm} \times 15 \mathrm{~mm}\right)$ at

$42537^{\circ} \mathrm{C}, 5 \% \mathrm{CO}_{2}$. After 3 days, the medium was removed; cells were treated with

$426 \quad 0.25 \%$ trypsin ( $3 \mathrm{ml}$ per petri dish) at $37^{\circ} \mathrm{C}$ for $5 \mathrm{~min}$ to allow cell dissociation. Then,

427 the cells were gently scraped from the plastic and resuspended cells in fresh

428 cDMEM for further assays.

429 PanB, panT, panDC, and monocytes were isolated from splenocytes of 8-12

430 wk B6 mice using EasySep Mouse B cell isolation kit (STEMCELL \#19854),

431 EasySep Mouse T cell isolation kit (STEMCELL \#19848), EasySep Mouse Pan-DC

432 enrichment kit (STEMCELL \#19763), and EasySep Mouse Monocyte isolation kit

433 (STEMCELL \#19861), respectively.

\section{In vitro BMDC stimulation}

$4351 \times 10^{5}$ cultured or isolated immune cells in $140 \mu$ cDMEM were placed in a 96-well 436 non-tissue culture treated microplate (Corning \#351172), mixed with $60 \mu$ of filter437 sterilized spent media (SSMs) for $48 \mathrm{hrs}$ incubation at $37^{\circ} \mathrm{C}, 5 \% \mathrm{CO}_{2}$, then

438 supplemented with zymosan at $4 \mu \mathrm{g} / \mathrm{ml}$ for additional $16 \mathrm{hrs}$ incubation under the 439 same condition. Finally, stimulated cultures were centrifuged at $500 \mathrm{~g}$ for $8 \mathrm{~min}$ to 440 harvest supernatants for the measurement of cytokines. To test the effect of ASF519

441 SSMs on TGF- $\beta$ production, no zymosan was added during the entire course (64 442 hrs) of stimulation of BMDCs. IL-10, TGF- $\beta$, and TNF- $\alpha$ were quantified by 443 corresponding ELISA kits (Invitrogen).

444 The impact on Treg proliferation was tested by co-culturing stimulated BMDCs 445 with splenocytes. We placed $1 \times 10^{6}$ BMDCs in $1 \mathrm{ml}$ cDMEM into a 24-well non446 tissue culture treated plate (Corning \#351147), stimulated cells with $0.5 \mathrm{ml}$ of 447 bacterial SSMs or $\mathrm{BHI}$ broth for 2 days at $37^{\circ} \mathrm{C}$ in the $\mathrm{CO}_{2}$ chamber, then further 448 stimulated cells with $4 \mu \mathrm{g} / \mathrm{ml}$ zymosan for additional $16 \mathrm{hrs}$ under the same condition. 449 Fresh splenocytes from $129 \mathrm{~S} 1$ mice were diluted to $1 \times 10^{\wedge} 6 \mathrm{cells} / \mathrm{ml}$ in phosphate450 buffered saline (PBS), stained with Celltrace violet (Thermo fisher \#C34571) at 10 $451 \mu \mathrm{M}, 37^{\circ} \mathrm{C}$ for $20 \mathrm{~min}$, washed once with five volumes of Gibco RPMI1640 medium 452 (Thermo fisher \#61870127), finally resuspended in RPMI1640 at $2 \times 10^{\wedge} 6 \mathrm{cell} / \mathrm{ml}$. To 453 coculture BMDCs and splenocytes, we removed the supernatants from stimulated 454 BMDCs by centrifugation at $500 \mathrm{~g}$ for $8 \mathrm{~min}$, resuspended cell pellets in $0.5 \mathrm{ml}$ 455 RPMI1640, then added $0.5 \mathrm{ml}$ of stained splenocytes for 4 days incubation in the 
$456 \mathrm{CO}_{2}$ incubator at $37^{\circ} \mathrm{C}$. Treg proliferation was analyzed by flow cytometry for co-

457 cultured cells stained by mouse CD4 and Foxp3 antibodies.

\section{Flow cytometry}

459 We generated single-cell suspensions from mouse colonic lamina propria tissue

460 using the Lamina Propria dissociation kit (Miltenyi Biotec \#130-097-410) by following

461 manufacturers' instruction. The program $37^{\circ} \mathrm{C} \_\mathrm{m} \_$LPDK_1 on the gentleMACS Octo

462 dissociator with heaters was used for tissue dissociation and cell extraction. Spleens

463 were harvested and gently grinded with the rubber head of syringe plunge through

$46470 \mu \mathrm{m}$ cell strainers to obtain signal cell suspension; red blood cells were then lysed

465 with the RBC lysis buffer (Invitrogen \#00-4333-57). Cells were preserved in the

466 CryoStor CS10 buffer (STEMCELL \#07930) for storage at $-80^{\circ} \mathrm{C}$.

$467 \quad$ Single-cell suspensions were stained and analyzed with BD LSRFortessa.

468 The panel included fluorescence conjugated antibodies against CD45, CD4, CD19,

469 CD11b, CD11c, CD103, F4/80, Foxp3, and IL-10. Cells were firstly stained by the

470 Zombi Violet Fixable Viability Kit, incubated with the FcR blocking reagent (anti-

$471 \mathrm{CD} 16 / C D 32$, BioLegend \#101302) in the cell staining buffer (BioLegend \#420201) at

472 room temperature for $10 \mathrm{~min}$, and then stained for surface markers, including CD45,

473 CD4, CD19, CD11b, CD11c, CD103, and F4/80. Stained cells were then fixed and

474 permeabilized by using the Foxp3 Fix/Perm buffer kit (BioLegend \#421403).

475 Permeabilized cells were finally stained for intracellular and nucleus markers. Cells

476 were acquired with a BD LSRFortessa, and analysis was performed with the FlowJo

477 software. Frequencies of each cell type were averaged for each experimental

478 condition.

$479 \quad$ For flow cytometry analysis of co-cultured cells, the panel included

480 fluorescence conjugated antibodies against CD4, CD8, and Foxp3. Cells were

481 stained with the APC eFluo 780 live/dead dye and then corresponding antibodies by

482 following the same staining protocol used for colonic lamina propria cells.

\section{Low input RNA-Seq}

4841,000 BMDC cells were collected directly into $5 \mu \mathrm{l} \mathrm{TCL}$ lysis buffer (Qiagen) with $1 \%$

485 2-mercaptoethanol. Lysates were frozen after $5 \mathrm{~min}$. Smart-seq2 libraries were

486 prepared as previously described ${ }^{37}$ and sequenced using the Broad Genomics

487 Platform. Reads were aligned to the mouse genome (GENCODE GRCm38/mm10 
primary assembly and gene annotation version M16;

489 https://www.gencodegenes.org/mouse/release_M16). The ribosomal RNA gene 490 annotations were removed from the general transfer format (GTF) file. The gene-

491 level quantification was calculated by featureCounts

492 (http://subread.sourceforge.net/). Raw read count tables were normalized by the 493 median of ratios method with the DESeq2 package from Bioconductor and then 494 converted to GenePattern GCT and CLS format. Samples with less than 3 million 495 uniquely mapped reads were automatically excluded from normalization to mitigate 496 the effect of samples with poor quality on normalized counts. Normalized read 497 counts were filtered for robust expression $(>10)$ to avoid confounders from low-level 498 noise and processed in the Multiplot suite and Morpheus

499 (https://software.broadinstitute.org/morpheus/). PCA was done using the prcomp 500 function in $\mathrm{R}$ on all genes.

\section{Metagenomic data analysis}

502 The genomic DNA was extracted from feces or cecal contents using the

503 ZymoBIOMICS DNA kit (Zymo \#D6300). Shotgun metagenomic sequencing was

504 performed using Illumina HiSeq2000 NextGen Sequencer. Data was cleaned by 505 removing mouse genome contamination. Microbial abundance was analyzed by 506 MetaPhIAn2 ${ }^{38}$. The prevalence of Parabacteroides goldsteinii in the human gut 507 microbiome studies was analyzed by the curatedMetagenomicData package ${ }^{39}$ in R.

\section{Bioassays for treated or fractionated samples}

509 To perform acetone precipitation, we mixed 4 times the sample volume of cold ($51020^{\circ} \mathrm{C}$ ) acetone to ASF519 SSMs or medium broth in an acetone-compatible bottle

511 and then incubated the mix for at least $60 \mathrm{~min}$ at $-20^{\circ} \mathrm{C}$. The precipitate was

512 collected by centrifugation at $13,000 \mathrm{~g}$ for $10 \mathrm{~min}$, then air-dried at room temperature

513 for 30 min before adding 1 x PBS buffer to dissolve the protein pellet on ice. Protein

514 concentration was measured by the Pierce BCA protein assay kit (Thermo Fisher

515 \#23227). We then evaluated the IL-10 inducing capability of acetone precipitates at 516 varying working concentrations in BMDC assays. To verify the role of proteinaceous

517 components in IL-10 induction and Treg proliferation, we treated SSMs of ASF519

518 grown in either the defined medium or supplemented $\mathrm{BHI}$ with immobilized

519 proteinase $\mathrm{K}$ to destroy proteins. In detail, we added $2 \mathrm{ml}$ of $33 \mathrm{mM}$ Tris- $\mathrm{HCl} \mathrm{pH} 7.5$ 
to reconstitute $10 \mathrm{U}$ protease-agarose power (1U/200 $\mu \mathrm{l}$, Sigma \#P9290-10UN),

521 mixed $1 \mathrm{ml}$ protein sample with $200 \mu \mathrm{l}$ of reconstituted protease, killed-protease

522 (generated by heating at $95^{\circ} \mathrm{C}$ for $10 \mathrm{~min}$ ), or $33 \mathrm{mM}$ Tris- $\mathrm{HCl} \mathrm{pH7.5,} \mathrm{for} \mathrm{incubation}$

523 at $37^{\circ} \mathrm{C}$ for $1 \mathrm{hr}$, followed by centrifugation to remove protease-agarose beads. By

524 following the in vitro BMDC stimulation protocol described above, we evaluated the

525 IL-10 inducing capability of heat- and proteinase $\mathrm{K}$ treated samples.

For gel filtration to fractionate proteins by size, we loaded the concentrated crude samples, following filtration using $0.2 \mu \mathrm{m}$ syringe filters, to the AKTA pure system with a $10 \mathrm{ml}$ sample injection loop and a HiLoad 16/600 Superdex 200 pg column. Proteins were eluted into $1 \times$ PBS at $1 \mathrm{ml} / \mathrm{min}$ and collected every $2 \mathrm{ml}$ in sterile microtubes. We then ran in vitro BMDC assays using $10 \mu$ of each fraction as the stimulus to determine bioactive fractions that can induce IL-10 production. The bioactive fractions were pooled and concentrated for further analysis, including protein quantification, native-/SDS-PAGE analysis, and protein identification by mass spectrometry. The gel filtration standard (Bio-Rad \#1511901) was analyzed with the same gel filtration settings to indicate the molecular size.

\section{Pharmacological inhibition}

537 To test whether ASF519 mediated IL-10 induction occurs via any of the adenosine 538 receptors, we employed the AHCY inhibitor 3-deazeadenosine (DZA, Sigma \#D8296-5MG) to block the formation of adenosine, the A1 antagonist DPCPX (Tocris \#0439), the A2a antagonist ZM 241385 (Thermo fisher \#10-361-0) and the A2b antagonist MRS-1754 (Tocris \#2752), or the A3 antagonist MRS-1523 (Sigma \#M1809-5MG). Stocks of antagonists were prepared in 50\% DMSO. We treated $1 \mathrm{x}$ $10^{5}$ BMDCs in $140 \mu \mathrm{l}$ with $2 \mu \mathrm{l}$ of a series of diluted antagonists or vehicle $(50 \%$

$544 \mathrm{DMSO}$ ) at $37^{\circ} \mathrm{C}$ for $30 \mathrm{~min}$, then added $60 \mu \mathrm{l}$ of broth control, ASF519 SSMs, or

545 protein extracts from SSMs to stimulate BMDCs for $48 \mathrm{hrs}$ in the $\mathrm{CO}_{2}$ chamber,

546 followed by further stimulation with zymosan at $4 \mu \mathrm{g} / \mathrm{ml}$ for $16 \mathrm{hrs}$ at $37^{\circ} \mathrm{C}$ in the $\mathrm{CO}_{2}$

547 chamber. Finally, we centrifuged the cell culture to collect supernatants for IL-10

548 ELISA assays.

549 The impact of TNF signaling on IL-10 induction of BMDCs was tested by 550 blocking the TNF signaling pathway with Etanercept ${ }^{40}$ (Sigma-Aldrich \#Y0001969) in 551 the BMDC assay using ASF519 SSMs and zymosan for step-wise stimulation. The 
552 levels of IL-10 and TNF-a in the BMDC supernatants were measured by ELISA kits 553 (Invitrogen).

\section{Expression of His-tagged proteins}

555 The coding genes of methionine adenosyltransferase (MAT), adenosyl

556 homocysteinase (AHCY), O-acetylhomoserine sulfhydrylase (METY), methionine

557 synthase (MS), O-acetylserine sulfhydrylase (OASS, also named cysteine synthase)

558 predicted in the ASF519 genome were cloned in the pET21(+) plasmid. Constructs

559 verified by Sanger sequencing were introduced into NEB Lemo21 DE3 for inducible

560 expression of His-tagged proteins. Bacterial cells were grown at $37^{\circ} \mathrm{C}$ in $\mathrm{LB}$ with 30

$561 \mu \mathrm{g} / \mathrm{ml}$ chloramphenicol, $50 \mu \mathrm{g} / \mathrm{ml}$ carbenicillin, and 0.5-1.5 mM L-rhamnose to

562 OD600 0.8, then 0.4 mM IPTG was added to induce protein expression for $20 \mathrm{hrs}$ at

$56318^{\circ} \mathrm{C}$. The recombinant enzymes were purified sequentially with a nickel affinity

564 column and a HiLoad 16/600 superdex 200pg column, in order to remove endotoxin

565 and get high-purity proteins. The purity of fractions in PBS was determined by SDS-

566 PAGE. High-purity fractions were pooled and concentrated with Amicon Ultra-15 10K

567 centrifugal filters, then mixed with an equal volume of $50 \%$ glycerol before protein

568 quantification with the BCA kit and storage at $-20^{\circ} \mathrm{C}$. To verify the adenosine forming

569 enzymatic activity, $1 \mu \mathrm{M}$ of all pure enzymes were pre-incubated with $1 \mathrm{mM} \beta-\mathrm{NAD}^{+}$

570 cofactor at $37^{\circ} \mathrm{C}$ for $30 \mathrm{~min}$, then supplemented with the substrate SAH at $1.67 \mathrm{mM}$

571 for incubation at $37^{\circ} \mathrm{C}$ for $10 \mathrm{~min}$. The level of adenosine was then quantified by the

572 adenosine fluorometric kit (Cell Biolabs \#MET-5090). Reactions with PBS replacing

573 pure enzymes served as the control. Each condition was performed in 3 replicates.

574 The impact of pure enzymes on IL-10 induction was evaluated by adding pure

575 enzymes into BMDC assays with $1 \times 10^{5}$ cells in $200 \mu \mathrm{l}$ in a 96-well microplate by

576 following the in vitro step-wise stimulation protocol. The level of IL-10 in cell culture

577 supernatants was determined by the IL-10 ELISA kit.

\section{Histological analyses}

579 We performed hematoxylin and Eosin (H\&E) staining to examine infiltration in

580 pancreatic islets. The slides were deparaffinized in two washes of xylenes at $5 \mathrm{~min}$

581 and then rehydrated in series of decreasing Ethanol concentration, 100\%, 95\%,

$58270 \%$, and $50 \%$ and distilled water. The slides were stained with hematoxylin (Gill-3,

583 Fisher \#3537-32) for 2 min and raised in running distilled water. The slides were 
584

585

586

587

588

589

590

591

592

593

594

595

596

597

598

599

600

601

602

603

604

605

606

607

608

609

610

611

612

613

614

615

616

617

destained (3-4 dips) with acid water and raised with distilled water then blued with ammonia water. After washing with distilled water and a quick $95 \%$ ethanol wash, the slides were stained with eosin (Thermo Scientific \#6766007) and dehydrated with two washes of $95 \%$ ethanol, two washes of $100 \%$ ethanol, and two washes of xylene. The slides were mounted with permount (Fisher \# SP15-500), cover with a coverslip, and left to dry overnight. Images were taken with EVOS $®$ FL Auto Imaging System (Life Technologies) at 20x in brightfield. The severity of insulitis in islets was assessed by bind pathological scoring.

To confirm the insulin producing function of islets, we conducted immunohistochemical staining of insulin in pancreas. The slides adjacent to those used in H\&E staining were deparaffinized in xylenes at 14 min and then rehydrated in series of decreasing ethanol concentration, $100 \%$ for $8 \mathrm{~min}, 95 \%$ for 6 minutes, and $70 \%$ for $10 \mathrm{~min}$ then in distilled water for $5 \mathrm{~min}$. The slides were washed with PBS w/ 0.2\% lamb serum for $5 \mathrm{~min}$ and then outlined with a PAP pen. The slides were blocked with Avidin (Vector Laboratories, \#SP-2001) for 30 minutes, washed with PBS w/ 0.2\% lamb serum for $5 \mathrm{~min}$, and then blocked with Biotin (Vector Laboratories, \#SP-2001) for 30 min and washed again with PBS w/ 0.2\% lamb serum. The slides were blocked with goat serum (Vector Laboratories, \#PK-4007) for $1 \mathrm{hr}$ and incubated overnight at $4^{\circ} \mathrm{C}$ with an anti-Insulin antibody (ab195956, 1:1000). The slide was warmed to room temperature for $1 \mathrm{hr}$ and washed with PBS $\mathrm{w} / 0.2 \%$ lamb serum for $5 \mathrm{~min}$ and then incubated with a biotinylated secondary antibody (Vector Laboratories, \#PK-4007) for one hour and washed PBS w/ 0.2\% lamb serum for $5 \mathrm{~min}$. Then the slides were incubated with the $A B C$ reagent (Vector Laboratories, \# PK-4007) for $1 \mathrm{hr}$ and washed with PBS. The slides were incubated in a peroxidase substrate solution (Vector Laboratories, \#SK-4105) for 2 min and wash with distilled water and counterstained with hematoxylin (Gill-3, Fisher \#353732) for $30 \mathrm{sec}$, and raised in running distilled water. The slides were then blued with ammonia water for $3 \mathrm{~min}$ and raised in running distilled waters. The slides were dehydrated with a series of washes with $70 \%$ ethanol for 5 minutes, $95 \%$ ethanol for $2 \mathrm{~min}, 100 \%$ ethanol for $2 \mathrm{~min}$, and xylene for $4 \mathrm{~min}$. The slides were mounted with permount (Fisher \#SP15-500), covered with a coverslip, and left to dry overnight. Images were taken with EVOS® FL Auto Imaging System (Life Technologies) at 20x in brightfield. We verified that the islets without insulitis still had functional beta cells by immunostaining for insulin. 


\section{Mouse Magnetic Luminex assays}

619 We performed a magnetic bead-based multiplex assay (R\&D systems \#LXSAMSM-

620 18) for the measurement of 18 analytes in sera, including BAFF/BLyS, IFN-gamma,

621 IL-1beta, IL-3, IL-5, IL-10, IL-13, IL-17E, IL-33, GM-CSF, IL-1alpha, IL-2, IL-4, IL-6,

622 IL-12p70, IL-17A, IL-27, and TNF-alpha. Serum samples were analyzed with two

623 replicates in a 96-w plate by following the manufacturer's instructions. The plate was

624 read with Luminex 200.

\section{Metabolomics}

626 We extracted metabolites from approximately $50 \mathrm{mg}$ cecal contents or a single fecal

627 pellet for the analysis of untargeted metabolomics. In detail, we resuspended

$628 \mathrm{cecal} / \mathrm{fecal}$ samples in $2 \mathrm{ml}$ methanol in $8 \mathrm{ml}$ glass vials (VWR \#66011-085) and

629 added $4 \mathrm{ml}$ cold chloroform for vortex mixing and homogenization in an ultrasound

630 water bath for $5 \mathrm{~min}$. We then added $2 \mathrm{ml} \mathrm{LC}-\mathrm{MS}$ grade water consisting of $100 \mathrm{nM}$

631 pentanoic acid as the internal standard. After vortex mixing, vials were placed in

632 centrifuge tubes for centrifugation at 3,000 rpm for $10 \mathrm{~min}$. The aqueous phase was

633 transferred to new glass vials and dried with nitrogen. Metabolites were resuspended

634 in a volume of acetonitrile:water (1:1) based on the sample weight $(200 \mu \mathrm{l} / 100 \mathrm{mg})$.

635 Samples were run on a Thermo Fisher Orbitrap IDX mass spectrometer. $5 \mu$ I

636 of the extracted sample was injected onto a $5 \mu \mathrm{m}$ Zic-pHILIC column $(150 \times 2.1 \mathrm{~mm})$.

637 Solvent A contained $20 \mathrm{mM}$ ammonium carbonate and $0.1 \%$ ammonium hydroxide in

638 Water; solvent B contained $97 \%$ acetonitrile in water. A linear gradient was used to

639 separate the analytes: 100 to $40 \%$ solvent B (0 to $19 \mathrm{~min}$ ), 40 to $0 \%$ solvent B (19 to

$64028 \mathrm{~min}$ ), $0 \%$ solvent B (28 to $33 \mathrm{~min}$ ), 0 to $100 \%$ solvent B (33 to $36 \mathrm{~min}$ ), and re-

641 equilibration of the column with $100 \%$ solvent B (36 to $45 \mathrm{~min}$ ) at a flow rate of 0.2

$642 \mathrm{ml} / \mathrm{min}$ and a column temperature of $40^{\circ} \mathrm{C}$.

643 The mass spectrometry parameters were as follows: HESI + and -; for MS1:

644 switching polarity, 120,000 resolution, range 65-1000, 40\% RF lens, AGC 1.e5,

645 maximum IT $50 \mathrm{~ms}$, using internal calibration; for MS2: threshold at 2e4, dynamic

646 exclusion, AquirX targeted exclusion, AquirX targeted inclusion - ddMS2 with HCD

647 stepped $15,25,45$, detected in orbitrap at 30,000 resolution, cycle of $0.6 \mathrm{sec}$ with

648 MS2 of other if time, same parameters as the inclusion. A pooled sample was

649 created from all samples and used for MS-MS runs using AquirX. For each AquirX

650 run, a blank and the pool was run in MS1, then four runs of MS2 ID were performed. 
651 AquirX was run in pos and neg separately. Functional analysis of untargeted

652 metabolome data was performed with the online tool MetaboAnalyst $5.0^{41}$

653 (https://www.metaboanalyst.ca/), using the Mummichog algorithm (positive mode; 3

654 ppm; p-value cutoff=0.001) and selecting the Mus musculus KEGG pathways

655 containing at least 3 entries.

656 qPCR analysis

657 We homogenized intestinal tissues in $0.5 \mathrm{ml}$ of TRIZOL buffer using the Retsch

658 Mixer Mill MM400 with two zirconia beads at 25 frequency/sec for $10 \mathrm{~min}$. Then, 100

$659 \mu$ chloroform was added for mixing and incubation at room temperature for 3 min.

660 We centrifuged samples at $12,000 \mathrm{~g}, 4 \mathrm{C}$ for $15 \mathrm{~min}$, transferred $300 \mu \mathrm{l}$ of the aqueous

661 phase to RNase-free tubes in order to mix with $150 \mathrm{ul} 100 \%$ ethanol, then isolated

662 and eluted the total RNA with the NucleoSpin RNA XS kit. The quality of extracted

663 RNA was assessed by Agilent RNA chips. The RNA concentration was determined

664 by a NanoDrop spectrophotometer. The cDNA was synthesized by iScript Reverse

665 Transcription Supermix (Bio-Rad \#1708841) using $1 \mu \mathrm{g}$ total RNA as the template.

666 We then performed qPCR using PowerUp SYBR Green Master Mix (Thermo fisher

667 \#A25742) on the QuantStudio Dx Real-Time PCR Instrument. Three-step

668 amplification was applied for each primer set. There were two technical replicates for

669 each primer set. Data was analyzed with the delta-delta Ct method ${ }^{42}$, using the

670 mouse gapdh gene as the internal control.

671 For the assessment of bacterial abundance, genomic DNA was extracted

672 from mouse feces using the ZymoBIOMICS DNA kit (Fisher Scientific \#50-125-

673 1501), then quantified with the Quantifluor ONE dsDNA System (Promega \#E4870).

674 DNA was diluted to $10-50 \mathrm{ng} / \mu \mathrm{l}$ and then used as the template for qPCR using the

675 universal 16S rRNA primers or P. goldsteinii ASF519 specific primers. Data was

676 analyzed with the delta-delta Ct method ${ }^{42}$, using the universal $16 \mathrm{~S}$ rRNA as a

677 reference.

\section{Statistical analysis}

679 Mice at similar ages were randomly allocated into experimental groups. No statistical

680 methods were used to predetermine sample size. Prism software (GraphPad, v8.4.3)

681 was used for all statistical analysis, unless otherwise specified. Student t tests, non-

682 parametric Mann-Whitney U tests, and two-way ANOVA with Tukey's multiple 
684 statistical test, the following $p$ values were used: ${ }^{* * * *} p<0.0001,{ }^{* * *} p<0.001,{ }^{* *} p<$

$6850.01,{ }^{*} p<0.05$ and not significant (ns). All graphs show the mean with sd or sem

686 specified, with each dot representing a biological sample (unless otherwise

687 specified). In the figure legends, n numbers refer to biological replicates, unless

688 otherwise specified.

\section{Acknowledgements}

690 We thank F. Simao for providing the BEND3 cell line; A. Wood and E. Chun for 691 suggestions on Flow Cytometry; L. Bry and V. Yeliseyev at the Massachusetts Host-

692 Microbiome Center for GF mice; and all members of the Laboratory for their comments 693 during the course of these studies and input during preparation of the manuscript. T.X. 694 is supported by a Mary K. lacocca fellowship. This research was supported by the 695 American Diabetes Association (ADA) Pathway to Stop Diabetes Initiator Award \#1696 17-INI-13 (A.D.K.) and a Smith Family Foundation Award for Excellence in Biomedical 697 Research (A.D.K.) It was also supported by an NIH/National Institute of Diabetes and 698 Digestive and Kidney Diseases Diabetes Research Grant No. P30 DK036836 699 (Principal Investigator: George L. King).

\section{Author contributions}

701 T.X. conceptualized, designed and conducted most of the experiments, performed 702 data analysis and prepared the manuscript. M.W. provided bacterial spent media. 703 L.Z., L.P., and M.Z.I. helped with in vivo mouse experiments. M.S.C. helped with 704 RNA sequencing and data analysis. L.P. stained and imaged pancreas sections.

705 C.O.B. and A.D.K. provided discussion, data interpretation and help with statistical 706 analyses. A.D.K. designed the experiments, interpreted the data and prepared the 707 manuscript. All authors helped to write and edit the manuscript.

\section{Competing interests}

709 A.D.K. is a co-founder and scientific advisor to FitBiomics, Inc. The other authors 710 declare no competing interests. 
712 1. Kawamoto, S. et al. Foxp3+ T cells regulate immunoglobulin A selection and 713 facilitate diversification of bacterial species responsible for immune homeostasis. Immunity 41, 152-165 (2014).

2. Round, J. L. \& Mazmanian, S. K. The gut microbiota shapes intestinal immune responses during health and disease. Nat. Rev. Immunol. 9, 313-323 (2009).

3. Zhang, D. et al. Neutrophil ageing is regulated by the microbiome. Nature $\mathbf{5 2 5}$, 528-532 (2015).

4. Josefsdottir, K. S., Baldridge, M. T., Kadmon, C. S. \& King, K. Y. Antibiotics

5. Wymore Brand, M. et al. The altered Schaedler flora: Continued applications of a defined murine microbial community. ILAR J. 56, 169-178 (2015).

6. Lathrop, S. K. et al. Peripheral education of the immune system by colonic commensal microbiota. Nature 478, 250-254 (2011).

7. Ruff, W. E., Greiling, T. M. \& Kriegel, M. A. Host-microbiota interactions in immune-mediated diseases. Nat. Rev. Microbiol. (2020) doi:10.1038/s41579020-0367-2.

8. Postler, T. S. \& Ghosh, S. Understanding the holobiont: How microbial metabolites affect human health and shape the immune system. Cell Metab. 26, 110-130 (2017).

9. Elzinga, J., van der Oost, J., de Vos, W. M. \& Smidt, H. The use of defined microbial communities to model host-microbe interactions in the human gut. Microbiol. Mol. Biol. Rev. 83, (2019).

10. Atarashi, K. et al. Treg induction by a rationally selected mixture of Clostridia strains from the human microbiota. Nature 500, 232-236 (2013).

11. Tanoue, T. et al. A defined commensal consortium elicits CD8 T cells and anticancer immunity. Nature 565, 600-605 (2019).

12. Geva-Zatorsky, N. et al. Mining the human gut microbiota for immunomodulatory organisms. Cell 168, 928-943.e11 (2017).

13. Biggs, M. B. et al. Systems-level metabolism of the altered Schaedler flora, a complete gut microbiota. ISME J. 11, 426-438 (2017).

14. Collins, J., Borojevic, R., Verdu, E. F., Huizinga, J. D. \& Ratcliffe, E. M. Intestinal 
microbiota influence the early postnatal development of the enteric nervous system. Neurogastroenterol. Motil. 26, 98-107 (2014).

15. Ivanov, I. I. et al. Specific microbiota direct the differentiation of IL-17-producing T-helper cells in the mucosa of the small intestine. Cell Host Microbe 4, 337-349 (2008).

16. Geuking, M. B. et al. Intestinal bacterial colonization induces mutualistic regulatory T cell responses. Immunity 34, 794-806 (2011).

17. Mosconi, I. et al. Intestinal bacteria induce TSLP to promote mutualistic T-cell responses. Mucosal Immunol. 6, 1157-1167 (2013).

18. Hapfelmeier, S. et al. Reversible microbial colonization of germ-free mice reveals the dynamics of IgA immune responses. Science 328, 1705-1709 (2010).

19. Bouskra, D. et al. Lymphoid tissue genesis induced by commensals through NOD1 regulates intestinal homeostasis. Nature 456, 507-510 (2008).

20. Keilbaugh, S. A. et al. Activation of Reglllbeta/gamma and interferon gamma expression in the intestinal tract of SCID mice: an innate response to bacterial colonisation of the gut. Gut 54, 623-629 (2005).

21. Lavoie, S. et al. The Crohn's disease polymorphism, ATG16L1 T300A, alters the gut microbiota and enhances the local Th1/Th17 response. Elife 8, (2019).

22. Li, X. et al. Response of germ-free mice to colonization with O. formigenes and altered Schaedler flora. Appl. Environ. Microbiol. 82, 6952-6960 (2016).

23. Burrows, M. P., Volchkov, P., Kobayashi, K. S. \& Chervonsky, A. V. Microbiota regulates type 1 diabetes through Toll-like receptors. Proc. Natl. Acad. Sci. U. S. A. 112, 9973-9977 (2015).

24. Haskó, G., Linden, J., Cronstein, B. \& Pacher, P. Adenosine receptors: therapeutic aspects for inflammatory and immune diseases. Nat. Rev. Drug Discov. 7, 759-770 (2008).

25. Turley, S., Poirot, L., Hattori, M., Benoist, C. \& Mathis, D. Physiological $\beta$ cell death triggers priming of self-reactive $T$ cells by dendritic cells in a type-1 diabetes model. J. Exp. Med. 198, 1527-1537 (2003).

26. Ramakrishna, C. et al. Bacteroides fragilis polysaccharide A induces IL-10 secreting $B$ and T cells that prevent viral encephalitis. Nat. Commun. 10, 2153 (2019).

27. Verma, R. et al. Cell surface polysaccharides of Bifidobacterium bifidum induce 

the generation of Foxp3+ regulatory T cells. Sci Immunol 3, (2018).

28. Sun, M. et al. Microbiota-derived short-chain fatty acids promote Th1 cell IL-10 production to maintain intestinal homeostasis. Nature Communications vol. 9 (2018).

29. Chen, H. et al. A forward chemical genetic screen reveals gut microbiota metabolites that modulate host physiology. Cell 177, 1217-1231.e18 (2019).

30. Cohen, L. J. et al. Functional metagenomic discovery of bacterial effectors in the human microbiome and isolation of commendamide, a GPCR G2A/132 agonist. Proceedings of the National Academy of Sciences vol. 112 E4825-E4834 (2015).

31. Gnad, T. et al. Adenosine activates brown adipose tissue and recruits beige adipocytes via A2A receptors. Nature 516, 395-399 (2014).

32. Schulz, N. et al. Critical role for adenosine receptor $A 2 a$ in $\beta$-cell proliferation. Mol Metab 5, 1138-1146 (2016).

33. Pearson, J. A., Wong, F. S. \& Wen, L. The importance of the Non Obese Diabetic (NOD) mouse model in autoimmune diabetes. J. Autoimmun. 66, 76-88 (2016).

34. O'Kell, A. L. et al. Comparative pathogenesis of autoimmune diabetes in humans, NOD mice, and canines: Has a valuable animal model of type 1 diabetes been overlooked? Diabetes 66, 1443-1452 (2017).

35. Brand, D. D., Latham, K. A. \& Rosloniec, E. F. Collagen-induced arthritis. Nat. Protoc. 2, 1269-1275 (2007).

36. Trouplin, V. et al. Bone marrow-derived macrophage production. J. Vis. Exp. (2013).

37. Picelli, S. et al. Full-length RNA-seq from single cells using Smart-seq2. Nat. Protoc. 9, 171-181 (2014).

38. Truong, D. T. et al. MetaPhIAn2 for enhanced metagenomic taxonomic profiling. Nat. Methods 12, 902-903 (2015).

39. Pasolli, E. et al. Accessible, curated metagenomic data through ExperimentHub. Nat. Methods 14, 1023-1024 (2017).

40. Scallon, B. et al. Binding and functional comparisons of two types of tumor necrosis factor antagonists. J. Pharmacol. Exp. Ther. 301, 418-426 (2002).

41. Chong, J. et al. MetaboAnalyst 4.0: towards more transparent and integrative metabolomics analysis. Nucleic Acids Res. 46, W486-W494 (2018). 
812 42. Livak, K. J. \& Schmittgen, T. D. Analysis of relative gene expression data using real-time quantitative PCR and the $2^{-\triangle \Delta C T}$ method. Methods vol. $25402-408$ (2001).

815 
a
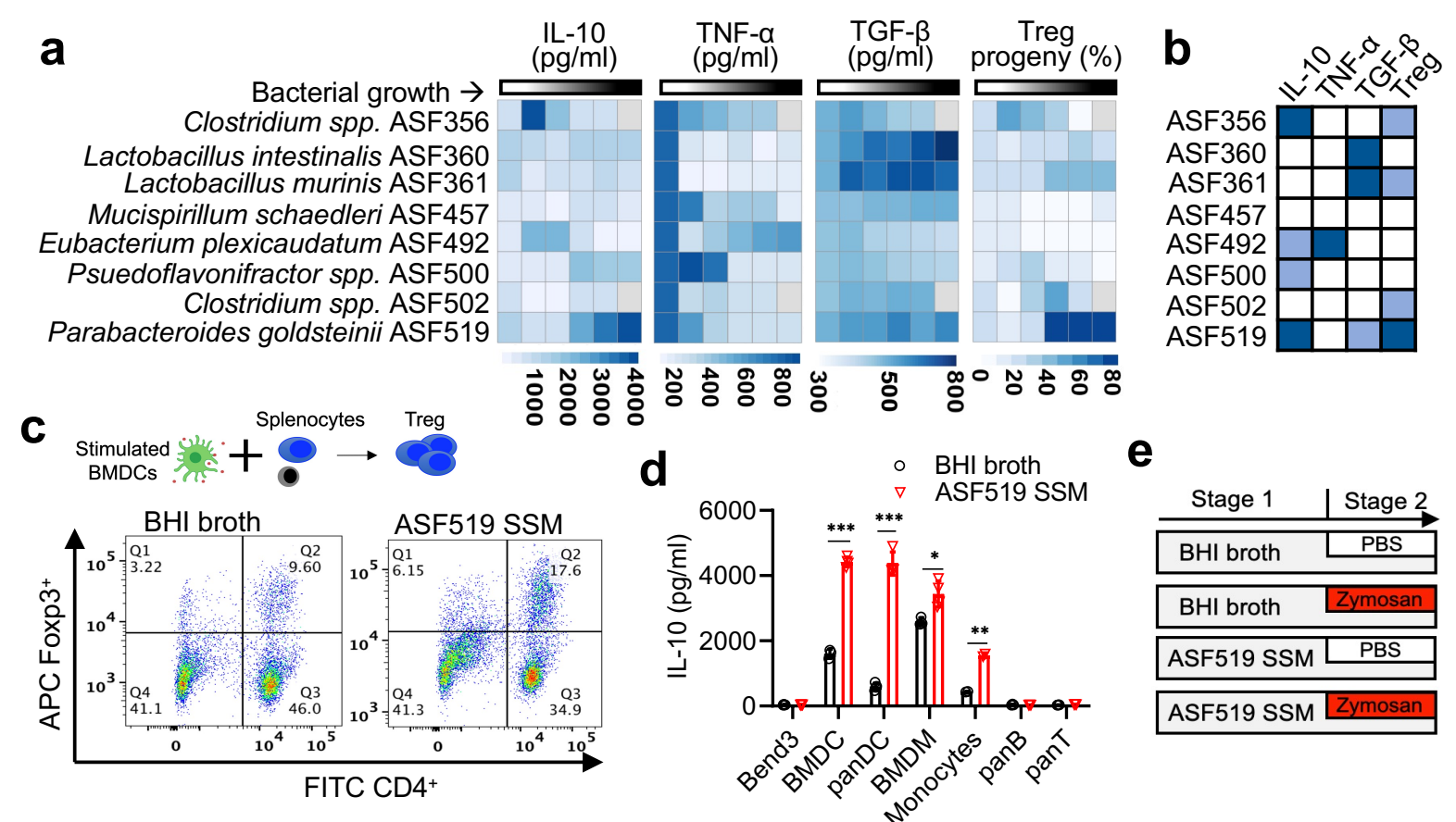

e

\begin{tabular}{|c|c|}
\hline Stage 1 & Stage 2 \\
\hline $\mathrm{BHI}$ broth & PBS \\
\hline BHI broth & Zymo \\
\hline ASF519 SSn & $\overline{\mathrm{PBS}}$ \\
\hline
\end{tabular}

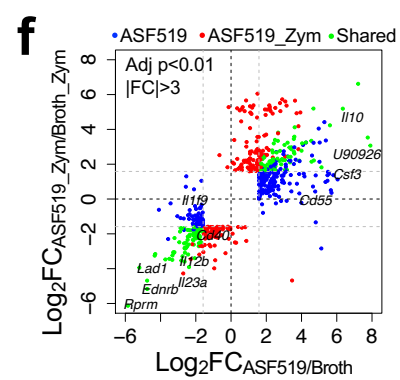

g
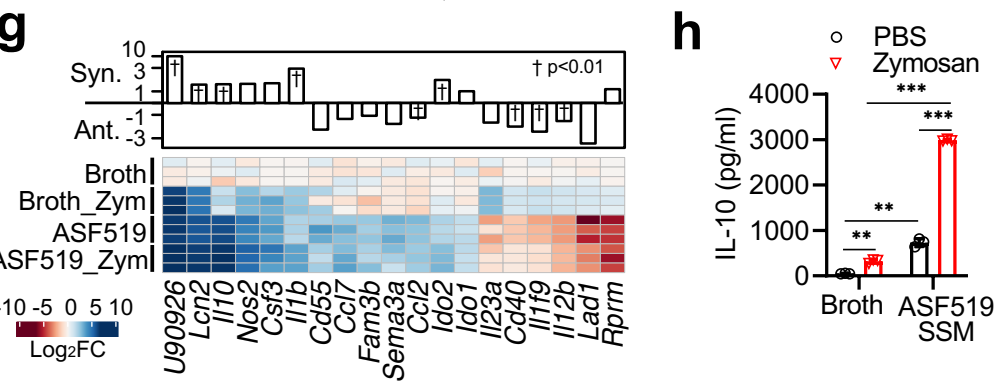

\section{Fig. 1 Parabacteroides goldsteinii ASF519 induces multifaced tolerogenic}

819 phenotypes. a, Measurement of cytokine production by BMDCs and Foxp3+ Treg

820 proliferation in the coculture of BMDCs and splenocytes, using filter sterilized spent

821 media collected during bacterial growth as the stimuli of BMDCs. These 8 bacterial

822 species are members of the altered Schaedler flora (ASF), a model of the murine gut

823 microbiome. Mean values of three biological replicates are shown. b. Prominent

824 immunophenotypes evoked by each ASF member irrespective of bacterial growth

825 stages. All blue cells present statistical significance $(p<0.05$, two-tailed Student $t$ test)

826 with at least a 35\% change in cytokine levels or a 2-fold change in Treg proliferation.

827 c, Flow cytometry analysis of Foxp3+ CD4+ T cells in splenocytes after coculture

828 with BMDCs stimulated by BHI broth or ASF519 SSM. Representative data are

829 shown $(\mathrm{n}=3)$. d, IL-10 production by cultured or enriched cell types after stimulation

830 with either broth or ASF519 SSM (n=3-4). e, Experimental design for RNA

831 sequencing of BMDCs that were exposed to bacterial spent medium and zymosan in 
832 a step-wise manner $(\mathrm{n}=3)$. $\mathbf{f}$, Intersection of differentially expressed genes (DEGs)

833 (adjusted $p<0.01$ and |fold change|>3) in two between-group comparisons, ASF519

834 versus Broth, and zymosan-involved ASF519_Zym versus Broth_Zym. g, Heatmap

835 of select DEGs in either comparisons shown in $\mathrm{f}$. The fold change of each gene was

836 relative to the Broth condition. The bar plot indicates fold changes in ASF519_Zym

837 versus ASF519, indicative of synergy (Syn.) and antagonism (Ant.) between ASF519

838 and zymosan. $\mathbf{h}, \mathrm{IL}-10$ production by BMDCs that underwent monotonous (bacterial

839 medium) or step-wise stimulation (bacterial medium + zymosan) $(n=3)$. Data (d, e,

840 and $\mathrm{h}$ ) are represented as mean+sd across biological replicates and analyzed by

841 two-tailed Student t test $\left({ }^{*} p<0.05 ;{ }^{* *} p<0.01 ;{ }^{* * *} p<0.001\right)$. 
a
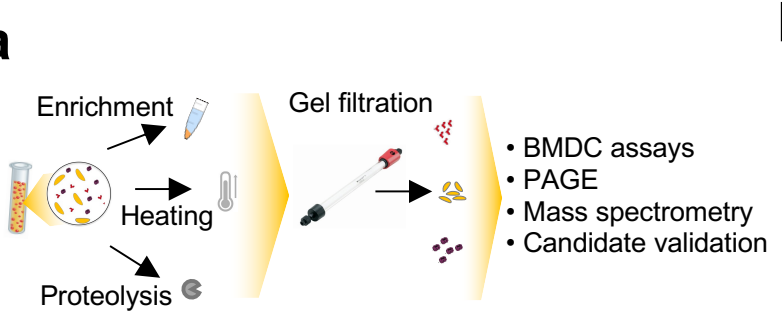

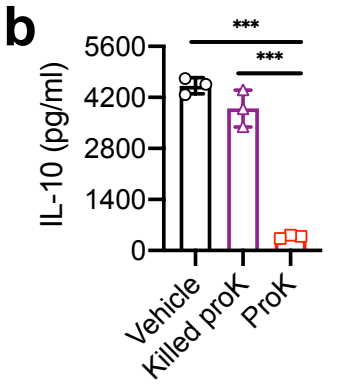

e

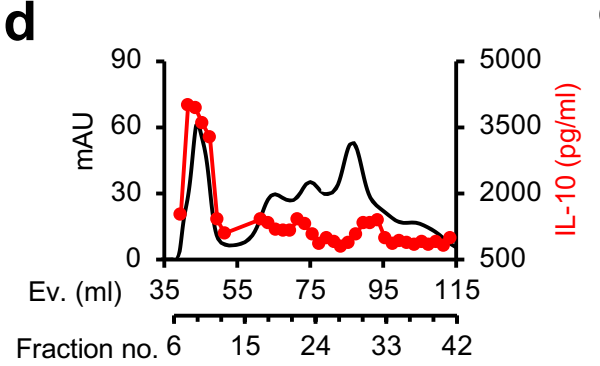

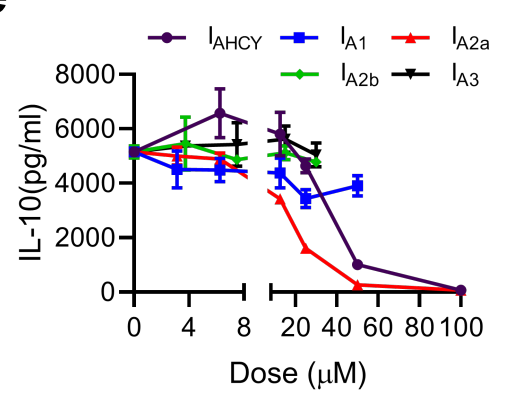

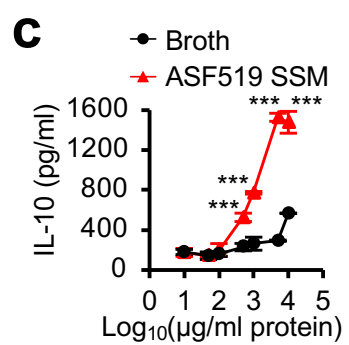

$\mathbf{f}$

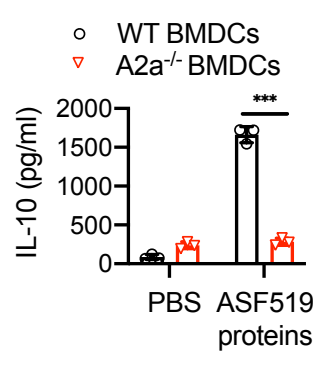

h $2000 \rightarrow$ MS $\rightarrow$ MAT $\rightarrow$ AHCY $\quad$ i

g

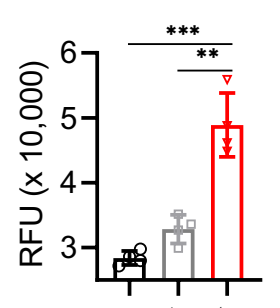

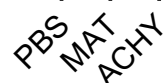

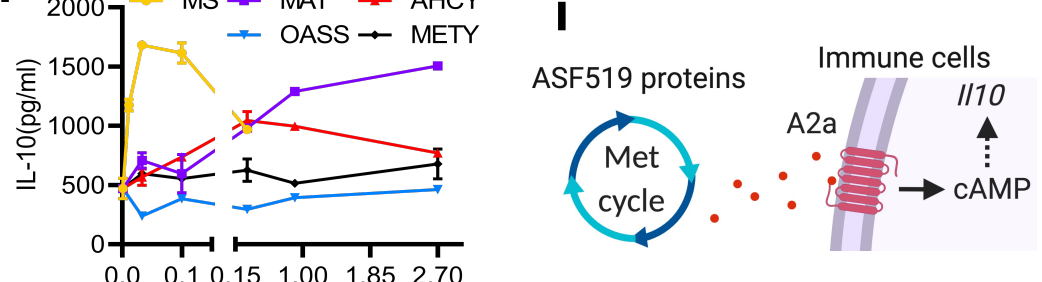

844

845 Fig. 2 Activation of adenosine receptor A2a by microbial enzymes enhances

846 immune tolerance. a, Bioanalytical strategy to identify responsible molecules in

847 bacterial spent media. b, Effect of immobilized protease K (ProK) treatment of

848 ASF519 SSM on IL-10 induction in BMDC assays $(n=3)$. The heat killed ProK and

849 ProK vehicle were used as controls. c, Dose-dependent induction of IL-10 by

850 enriched crude proteins in BMDC assays $(n=3) . \mathbf{d}, I L-10$ inducing function of each

851 fraction eluted from gel filtration of crude ASF519 SSM. The UV absorbance of

852 proteins (mAU) and IL-10 readouts were plotted against the elution volume (Ev.) and

853 elution fractions, colored by black and red, respectively. Data are representative of

854 reproducible runs. e, Effect of inhibition of adenosine forming enzyme AHCY and

855 adenosine receptors (A1, A2a, A2b, A3) by specific antagonists on IL-10 induction of

856 BMDCs in response to ASF519 SSM. f, Validation of A2a engagement in IL-10

857 induction. Wildtype (WT) and A2a $\mathrm{a}^{-/-}$BMDCs were stimulated by fractionated ASF519

858 proteins or PBS $(n=3)$. g, Adenosine formation by purified enzymes in in vitro assays

859 fed with the substrate S-adenosylhomocysteine $(n=4)$. MAT, S-adenosylmethionine 
860 synthase; AHCY, Adenosylhomocysteinase. h, IL-10 induction by purified enzymes

861 that are involved in methionine and cysteine metabolism $(n=3)$. MS, methionine

862 synthase; METY, O-acetyl-L-homoserine sulfhydrylase; OASS, O-acetylserine

863 sulfhydrylase. i, Model of ASF519-mediated regulation of IL-10 induction in BMDCs.

864 Enzymes of the methionine cycle interact with immune cells to activate the

865 adenosine A2a receptor signaling pathway to induce IL-10 production. Data (b, c, $f$

866 and g) are represented as mean+sd and analyzed by two-tailed Student $t$ test

$867 \quad\left({ }^{*} p<0.05,{ }^{* *} p<0.01 ;{ }^{* * *} p<0.001\right)$.

868 
a

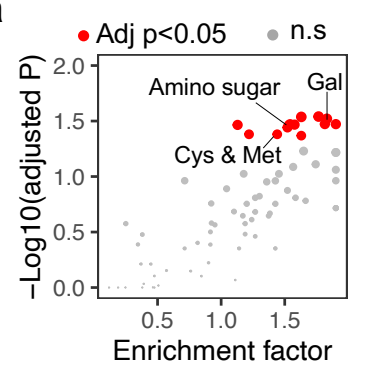

d

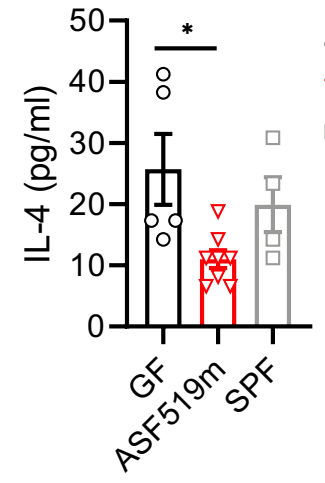

g

869

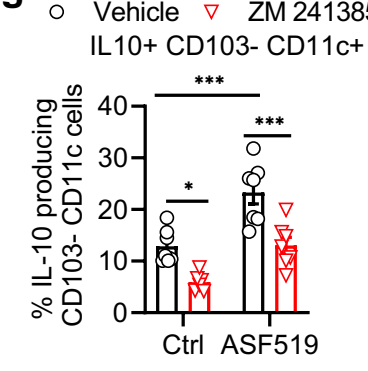

b
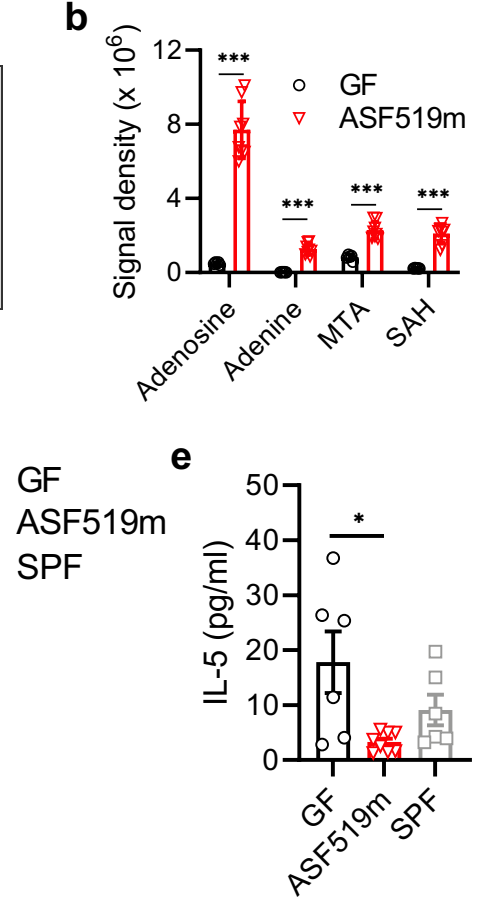

C

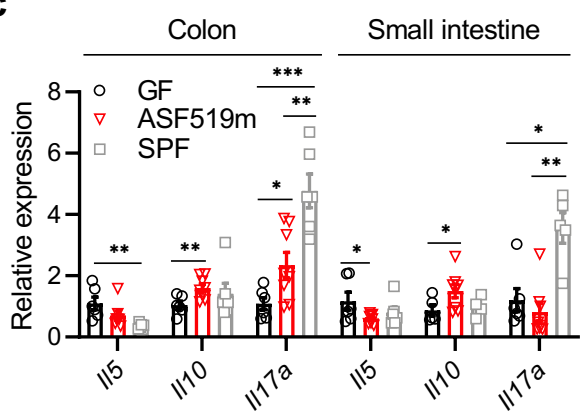

f

Oral gavage

(live vs killed ASF519)

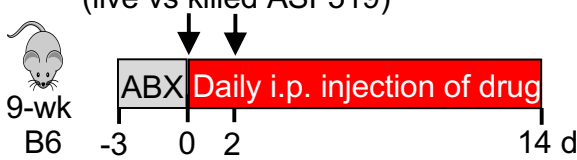

h

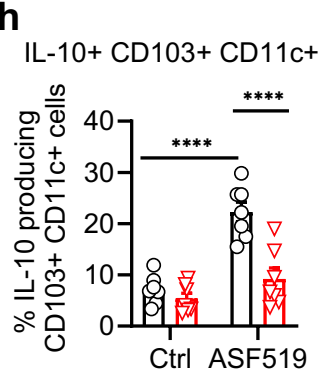

i

CD19+ CD45+ j

$\mathrm{CD} 11 \mathrm{~b}+\mathrm{CD} 45+$
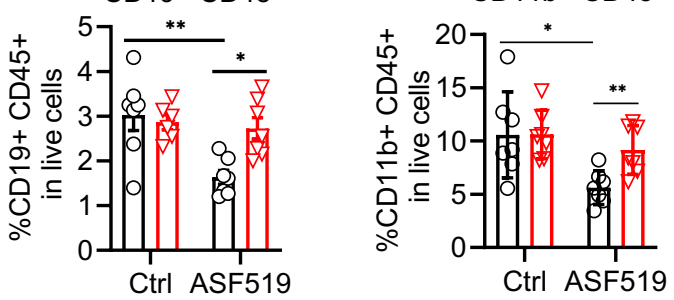

871 Fig. 3 P. goldsteinii ASF519 in mice alters the cecal metabolome and immune

872 phenotypes. a, Effect of ASF519 mono-association (ASF519m) in gnotobiotic mice

873 on metabolic pathways through functional analysis of cecal metabolome data. The

874 metabolism of 13 amino acids (e.g., Cys and Met), galactose (Gal), and amino and

875 nucleotide sugars were significantly influenced by ASF519m versus germ-free mice

876 (Extended Data Fig. 7b). b, Signal density of identified cecal metabolites. Data are

877 represented as mean+sem $(n=5-8)$ and analyzed by Mann-Whitney $U$ test

$\left.878 \quad{ }^{* * *} p<0.001\right) . c, q P C R$ assays on the transcript abundance of select genes in small

879 intestine and colon collected from GF, ASF519m, and SPF mice. Data are

880 represented as mean+sem $(n=5-8)$ and analyzed by two tailed Student $t$ test

$881 \quad\left({ }^{*} p<0.05 ;{ }^{* *} p<0.01 ;{ }^{* * *} p<0.001\right)$. $\mathbf{d}$ and $\mathbf{e}$, measurement of IL-4 (d) and IL-5 (e) in

882 sera of GF, ASF519m, and SPF mice. Data are represented as mean \pm sem ( $n=4-8)$

883 and analyzed by unpaired, nonparametric Mann-Whitney test, " $\mathrm{p}<0.05$. f,

884 Experimental design to test the role of adenosine receptor A2a in ASF519-mediated 
885 immunomodulation in antibiotic-treated ( $A B X)$ mice. Mice that received two doses of 886 live or killed ASF519 were intraperitoneally injected daily with the A2a antagonist, 887 ZM 241385. g-j, Flow cytometry analysis of IL-10 producing CD11c+ dendritic cells 888 ( $g$ and $h$ ), CD19+ CD45+ B cells (i), and CD11 $b+C D 45+$ cells $(j)$. Data are 889 represented as mean \pm sem $(n=6-8)$ and analyzed by two-way ANOVA along with 890 Tukey's test $\left({ }^{*} p<0.05 ;{ }^{* *} p<0.01 ;{ }^{* * *} p<0.001 ;{ }^{* * *} p<0.0001\right)$.

891 

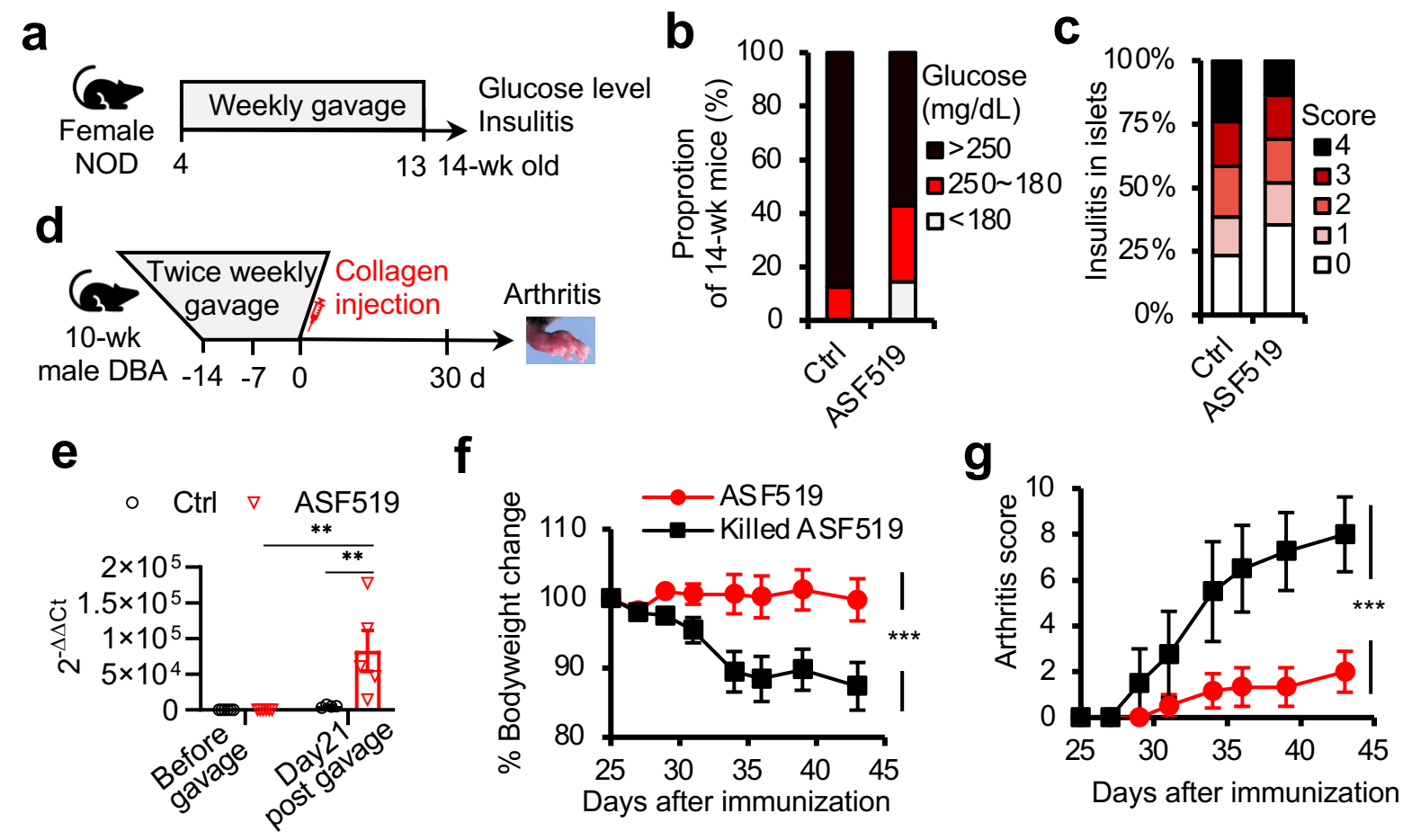

893

894 Fig. 4 P. goldsteinii ASF519 supplementation ameliorates disease severity in mouse models of type 1 diabetes (T1D) and collagen-induced arthritis (CIA). a,

896 Experimental design to test the effect of orally administered heat-killed (Ctrl) or live

897 ASF519 on T1D in female NOD mice. $\mathbf{b}$, The percentage of mice grouped by non-

898 fast blood glucose levels at 14-wk old ( $n=7-8)$. c, Histological insulitis scores of

899 pancreatic islets in Ctrl and ASF519 mice. Each group has at least 120 islets

900 assessed. The insulin producing function of insulitis free islets was validated by

901 immunohistochemical staining of insulin. d, Experimental design to test the role of

902 ASF519 in the collagen-induced arthritis mouse model. e, Comparison of ASF519

903 abundance in stools before gavage and 21 days post gavage by qPCR. Data are

904 represented as mean+sem $(n=5-6)$ and analyzed by Mann-Whitney $U$ test $\left({ }^{* *} p<0.01\right.$;

$\left.905{ }^{* * *} p<0.001\right)$. $\mathbf{f}$, Loss of body weight after immunization with collagen $(n=5-6) . \mathbf{g}$,

906 Arthritis severity developed on four paws of immunized Ctrl and ASF519 mice. Data

907 ( $f$ and $g$ ) are represented by mean+sem $(n=5-6)$ and the significance was tested by

908 two-way ANOVA $\left({ }^{* * *} p<0.001\right)$.

909

910 


\section{Figures}

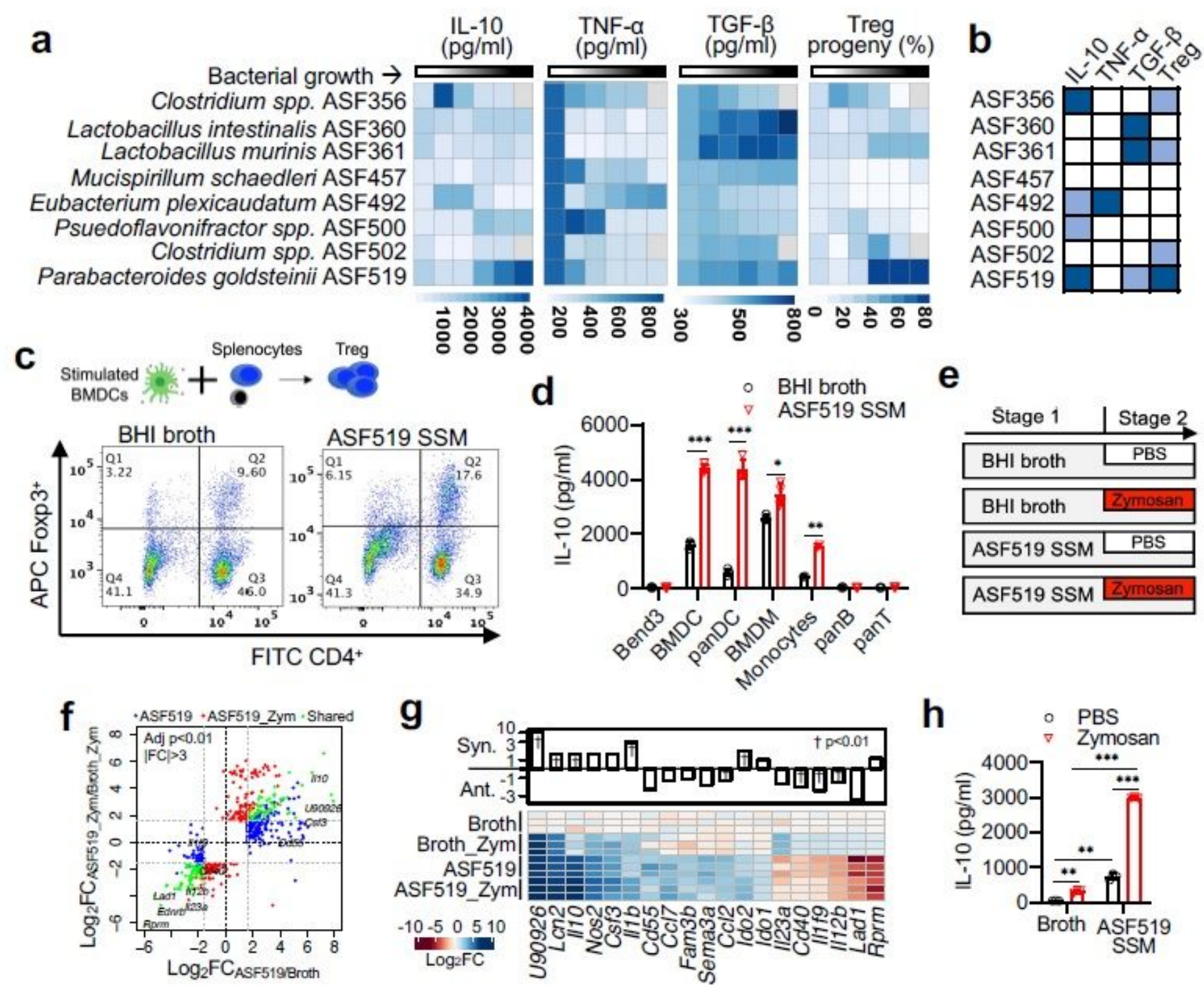

Figure 1

Parabacteroides goldsteinii ASF519 induces multifaced tolerogenic phenotypes. a, Measurement of cytokine production by BMDCs and Foxp3+ Treg proliferation in the coculture of BMDCs and splenocytes, using filter sterilized spent media collected during bacterial growth as the stimuli of BMDCs. These 8 bacterial species are members of the altered Schaedler flora (ASF), a model of the murine gut microbiome. Mean values of three biological replicates are shown. b, Prominent immunophenotypes evoked by each ASF member irrespective of bacterial growth stages. All blue cells present statistical significance ( $p<0.05$, two-tailed Student $t$ test) with at least a $35 \%$ change in cytokine levels or a 2 -fold change in Treg proliferation. c, Flow cytometry analysis of Foxp3+ CD4+ T cells in splenocytes after coculture with BMDCs stimulated by BHI broth or ASF519 SSM. Representative data are shown $(n=3)$. d, 
IL-10 production by cultured or enriched cell types after stimulation with either broth or ASF519 SSM ( $n=3-4)$. e, Experimental design for RNA sequencing of BMDCs that were exposed to bacterial spent medium and zymosan in a step-wise manner $(n=3)$. $f$, Intersection of differentially expressed genes (DEGs) (adjusted $p<0.01$ and |fold changel $>3$ ) in two between-group comparisons, ASF519 versus Broth, and zymosan-involved ASF519_Zym versus Broth_Zym. g, Heatmap of select DEGs in either comparisons shown in $\mathrm{f}$. The fold change of each gene was relative to the Broth condition. The bar plot indicates fold changes in ASF519_Zym versus ASF519, indicative of synergy (Syn.) and antagonism (Ant.) between ASF519 and zymosan. $\mathrm{h}, \mathrm{IL}-10$ production by BMDCs that underwent monotonous (bacterial medium) or step-wise stimulation (bacterial medium + zymosan) $(n=3)$. Data $(d, e$, and $h)$ are represented as mean+sd across biological replicates and analyzed by two-tailed Student $t$ test $\left({ }^{*} p<0.05 ;{ }^{* \star} p<0.01\right.$; $\star \star \star p<0.001)$.

a

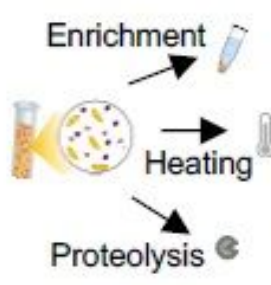

d

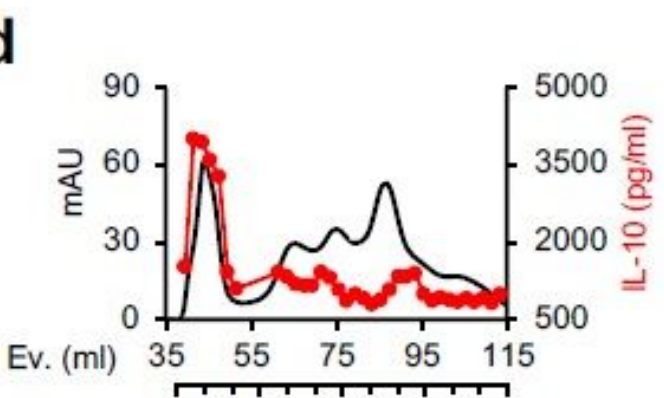

Fraction no. $6 \quad 15 \quad 24 \quad 33 \quad 42$
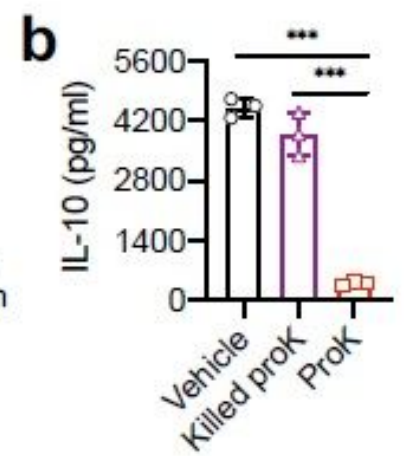

e

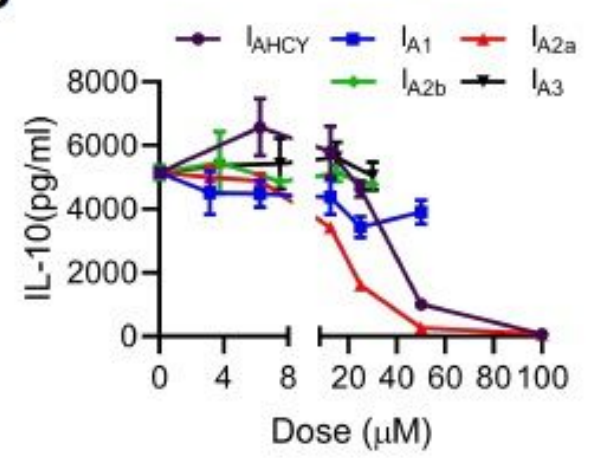

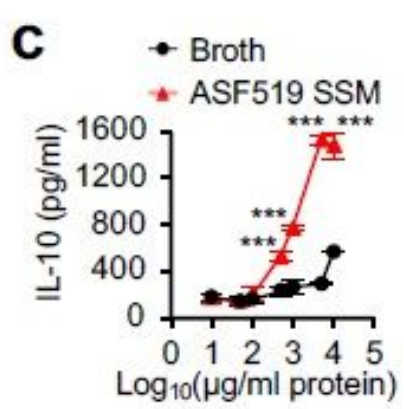

f

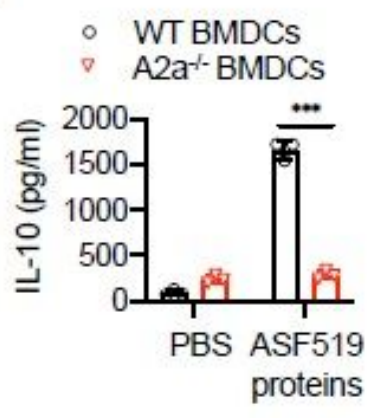

g

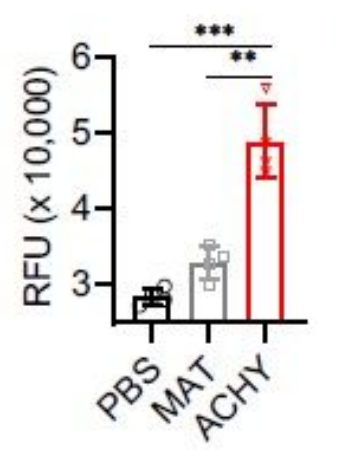

h

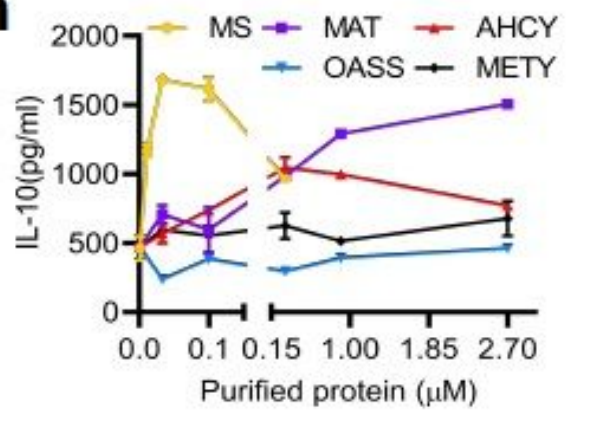

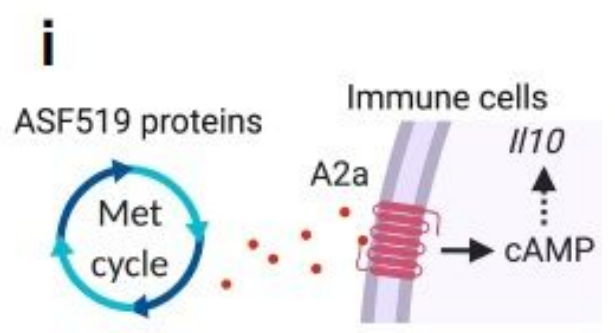

Figure 2

Activation of adenosine receptor A2a by microbial enzymes enhances immune tolerance. a, Bioanalytical strategy to identify responsible molecules in bacterial spent media. b, Effect of immobilized protease K 
(ProK) treatment of ASF519 SSM on IL-10 induction in BMDC assays $(n=3)$. The heat killed ProK and ProK vehicle were used as controls. c, Dose-dependent induction of IL-10 by enriched crude proteins in BMDC assays $(n=3)$. d, IL-10 inducing function of each fraction eluted from gel filtration of crude ASF519 SSM. The UV absorbance of proteins (mAU) and IL-10 readouts were plotted against the elution volume (Ev.) and elution fractions, colored by black and red, respectively. Data are representative of reproducible runs. e, Effect of inhibition of adenosine forming enzyme AHCY and adenosine receptors (A1, A2a, A2b, A3) by specific antagonists on IL-10 induction of BMDCs in response to ASF519 SSM. $f$, Validation of $A 2 a$ engagement in IL-10 induction. Wildtype (WT) and A2a-/- BMDCs were stimulated by fractionated ASF519 proteins or PBS ( $n=3)$. g, Adenosine formation by purified enzymes in in vitro assays fed with the substrate $S$-adenosylhomocysteine $(n=4)$. MAT, S-adenosylmethionine synthase; AHCY, Adenosylhomocysteinase. h, IL-10 induction 860 by purified enzymes that are involved in methionine and cysteine metabolism ( $n=3)$. MS, methionine synthase; METY, O-acetyl-L-homoserine sulfhydrylase; OASS, 0-acetylserine sulfhydrylase. i, Model of ASF519-mediated regulation of IL-10 induction in BMDCs. Enzymes of the methionine cycle interact with immune cells to activate the adenosine A2a receptor signaling pathway to induce IL-10 production. Data (b, c, $f$ and $g$ ) are represented as mean+sd and analyzed by two-tailed Student $t$ test $\left({ }^{*} p<0.05,{ }^{\star \star} p<0.01 ;{ }^{* \star \star} p<0.001\right)$. 
a

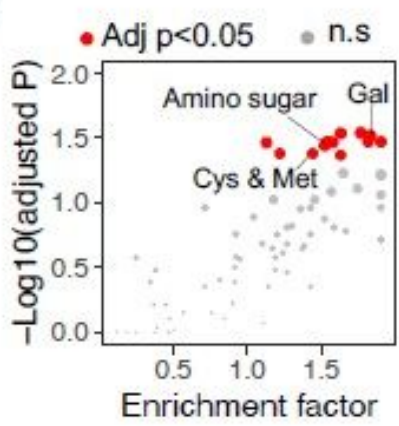

b

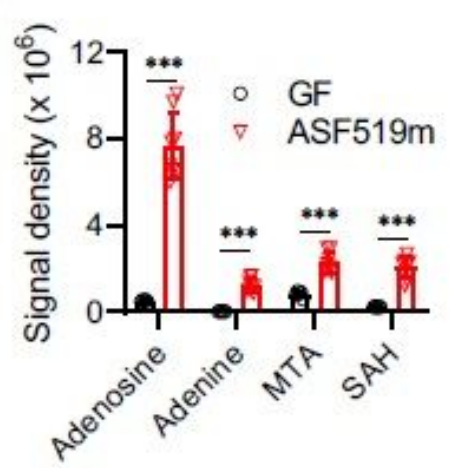

e

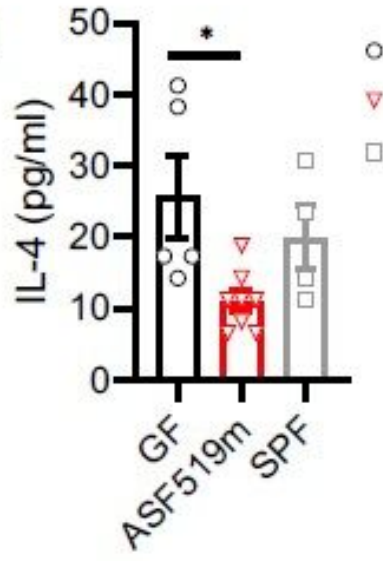

g

- Vehicle $\nabla$ ZM 241385 IL10+ CD103- CD11C+

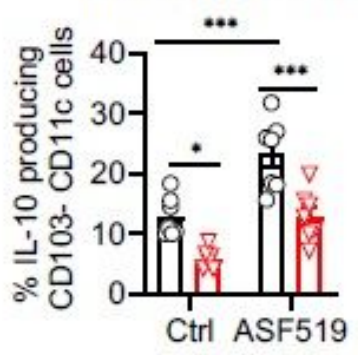

GF

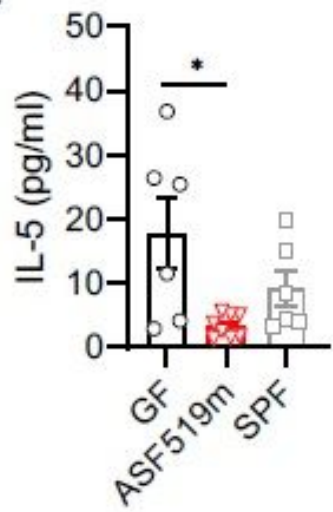

h

IL-10+ CD103+ CD11C+

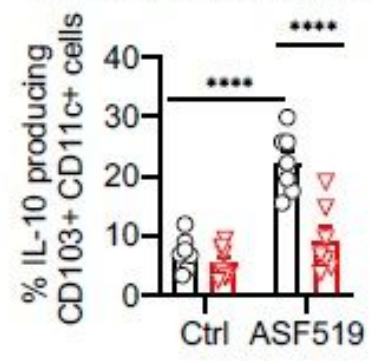

C

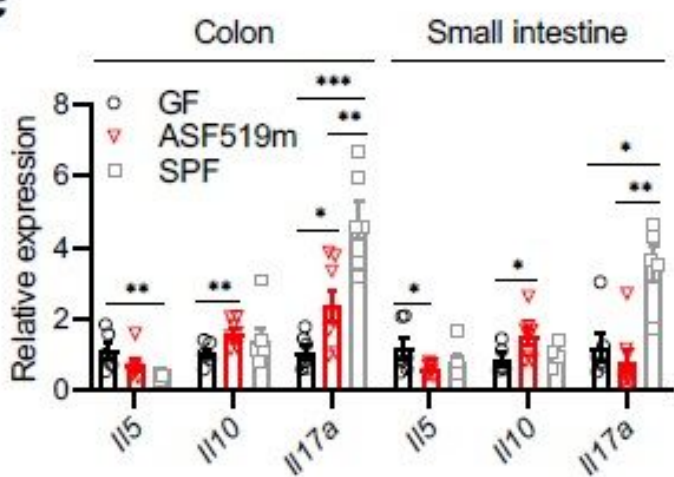

$\mathbf{f}$

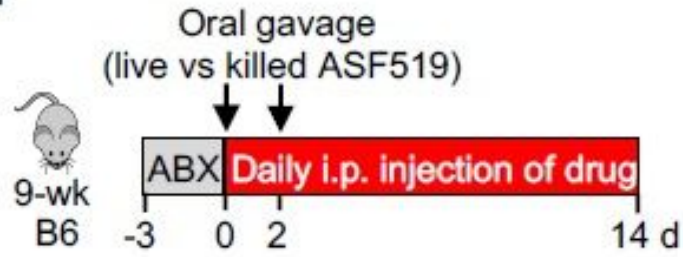

i

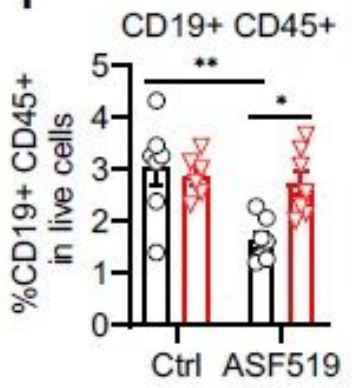

$\mathrm{CD} 11 \mathrm{~b}+\mathrm{CD} 45+$

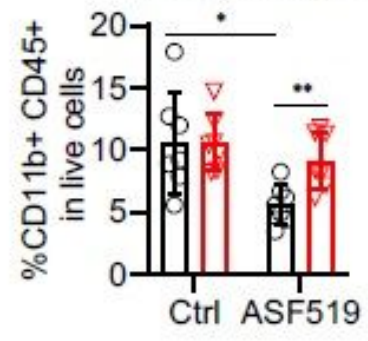

\section{Figure 3}

P. goldsteinii ASF519 in mice alters the cecal metabolome and immune phenotypes. a, Effect of ASF519 mono-association (ASF519m) in gnotobiotic mice on metabolic pathways through functional analysis of cecal metabolome data. The metabolism of 13 amino acids (e.g., Cys and Met), galactose (Gal), and amino and nucleotide sugars were significantly influenced by ASF519m versus germ-free mice (Extended Data Fig. 7b). b, Signal density of identified cecal metabolites. Data are represented as mean+sem $(n=5-$ 8) and analyzed by Mann-Whitney U test ( $* \star * p<0.001)$. c, qPCR assays on the transcript abundance of select genes in small intestine and colon collected from GF, ASF519m, and SPF mice. Data are represented as mean+sem $(n=5-8)$ and analyzed by two tailed Student test $\left({ }^{*} p<0.05 ;{ }^{* *} p<0.01\right.$; $\left.{ }^{* * *} \mathrm{p}<0.001\right)$. $d$ and e, measurement of IL-4 (d) and IL-5 (e) in sera of GF, ASF519m, and SPF mice. Data are represented as mean+sem $(n=4-8)$ and analyzed by unpaired, nonparametric Mann-Whitney test, ${ }^{\star} p<0.05$. $f$, Experimental design to test the role of adenosine receptor A2a in ASF519- 
mediated immunomodulation in antibiotic-treated ( $A B X)$ mice. Mice that received two doses of live or killed ASF519 were intraperitoneally injected daily with the A2a antagonist, ZM 241385. g-j, Flow cytometry analysis of IL-10 producing CD11c+ dendritic cells ( $g$ and $h$ ), CD19+ CD45+ B cells (i), and $\mathrm{CD} 11 \mathrm{~b}+\mathrm{CD} 45+$ cells $(\mathrm{j})$. Data are represented as mean+sem $(n=6-8)$ and analyzed by two-way ANOVA along with Tukey's test $\left({ }^{*} p<0.05 ;{ }^{* *} p<0.01 ; * * * p<0.001 ; * \star * * p<0.0001\right)$.
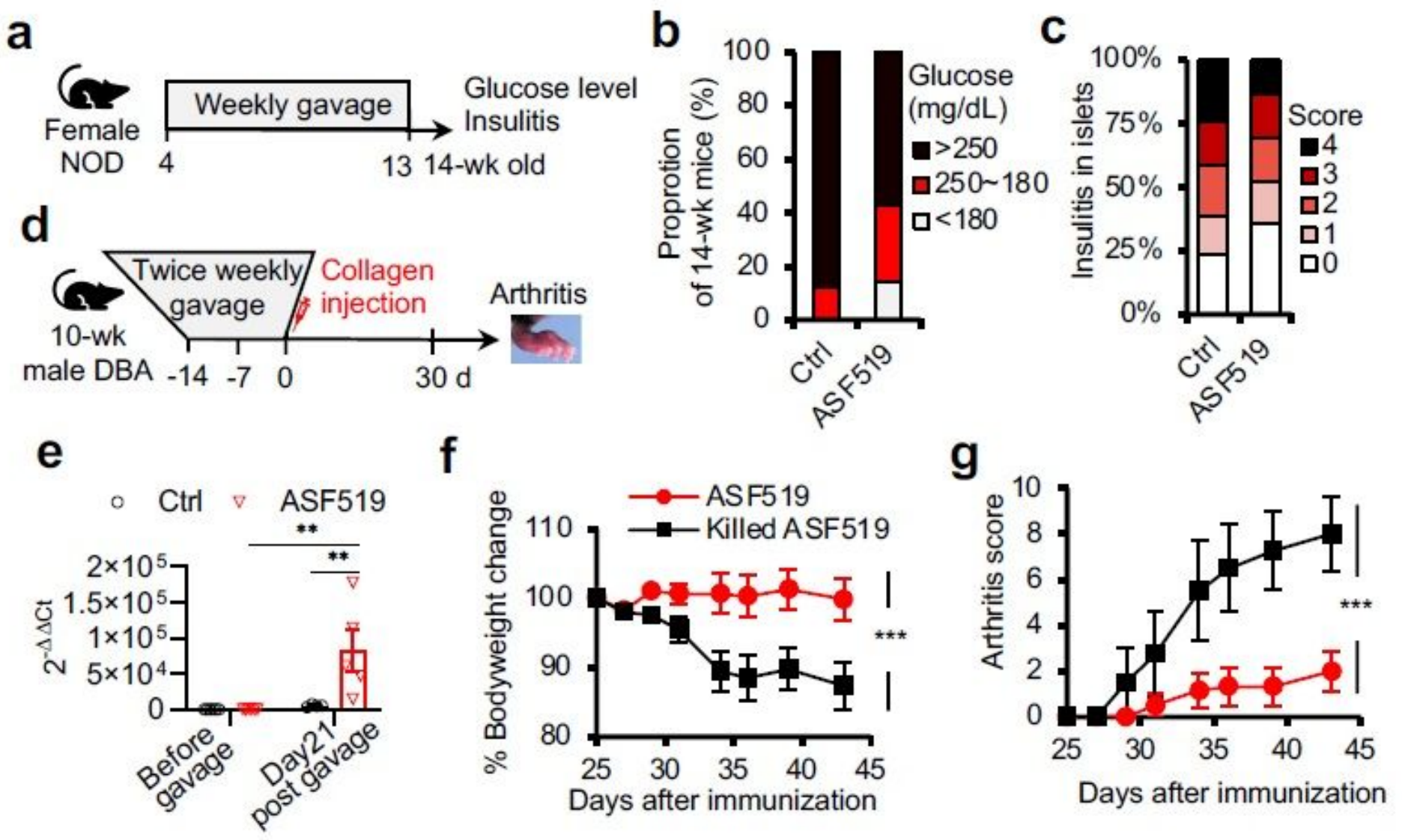

\section{Figure 4}

P. goldsteinii ASF519 supplementation ameliorates disease severity in mouse models of type 1 diabetes (T1D) and collagen-induced arthritis (CIA). a, Experimental design to test the effect of orally administered heat-killed (Ctrl) or live ASF519 on T1D in female NOD mice. b, The percentage of mice grouped by non fast blood glucose levels at 14-wk old $(n=7-8)$. c, Histological insulitis scores of pancreatic islets in Ctrl and ASF519 mice. Each group has at least 120 islets assessed. The insulin producing function of insulitis free islets was validated by immunohistochemical staining of insulin. $d$, Experimental design to test the role of ASF519 in the collagen-induced arthritis mouse model. e, Comparison of ASF519 abundance in stools before gavage and 21 days post gavage by qPCR. Data are represented as mean+sem $(n=5-6)$ and analyzed by Mann-Whitney U test $(* \star p<0.01 ; * \star * p<0.001) . f$, Loss of body weight after immunization with collagen ( $n=5-6)$. g, Arthritis severity developed on four paws of immunized Ctrl and ASF519 mice. Data ( $f$ and $g)$ are represented by mean+sem $(n=5-6)$ and the significance was tested by two-way ANOVA $\left({ }^{\star \star *} \mathrm{p}<0.001\right)$. 


\section{Supplementary Files}

This is a list of supplementary files associated with this preprint. Click to download.

- Extendeddatasubmitted03292021.pdf 